\title{
Transparenz und Kontrolle bei related party transactions: Verträge der Gesellschaft mit ihrem Aufsichtsratsmitglied
}

\begin{abstract}
Geschäfte der Gesellschaft mit einem Aufsichtsratsmitglied müssen dem Aufsichtsrat vorgelegt werden und bedürfen seiner Zustimmung. Damit sollen Interessenkonflikte vermieden und Transparenz hergestellt werden. Der Beitrag geht folgenden Fragen nach: Wen trifft die Pflicht zur Information? Wie genau ist der Aufsichtsrat über diese Geschäfte zu informieren? Welche Stimmverbote bestehen? Welche Konsequenzen können sich daraus für die Beschlussfähigkeit ergeben? Welche Rechtsfolgen löst die Nichtbeachtung der gesetzlichen Anforderungen aus? Schließlich wird die nicht ausreichend geregelte Situation der Arbeitnehmervertreter untersucht.
\end{abstract}

Deskriptoren: Zustimmungspflichtige Geschäfte; Aufsichtsratsvergütung; Beratungsvertrag; Konzerngeschäft, Corporate Governance; Vertretungsmacht des Vorstands; Interessenkonflikt; Stimmverbot; Zustimmungsvorbehalt; Arbeitnehmervertreter.

$\S 71$ Abs 1, § 87 Abs 1a, § 95 Abs 5, § 98 AktG; § 25 Abs 4, § 30j Abs 5 GmbHG; Regel 48 CGK.

\section{Übersicht:}

A. Problemstellung

B. Geschäfte mit Aufsichtsratsmitgliedern - welche Sachverhalte, welche Gefahren?

C. Vorbilder für $\S 95$ Abs 5 Z 12 AktG (und $\S 30 \mathrm{j}$ Abs 5 $\mathrm{Z} 10 \mathrm{GmbHG}$ )

D. Anwendungsbereich von $\S 95$ Abs $5 \mathrm{Z} 12$ AktG (und $\S 30 \mathrm{j}$ Abs $5 \mathrm{Z} 10 \mathrm{GmbHG})$

E. Rechtsfolgen

F. Unerledigte Probleme bei Arbeitnehmervertretern

G. Ausblick

\section{A. Problemstellung}

Aufsicht setzt voraus, dass die Aufseher von den $\mathrm{zu}$ beaufsichtigenden Personen nicht abhängig sind. Ähnlich verhält es sich mit der Beratung: Ratschläge werden nicht frei und unvoreingenommen erteilt, wenn der Ratgeber abhängig von dem Beratungsempfänger ist. Der Aufsichtsrat hat die Geschäftsführung zu überwachen $\left.{ }^{1}\right)$. Nach einhelliger Meinung gehört die Beratung des Vorstands in Fragen grundsätzlicher Bedeutung zu den Aufgaben des Aufsichtsrats ${ }^{2}$ ). Für die begleitende Überwachung künftiger Entwicklungen ${ }^{3}$ ) bedarf es der lau-

1) $\S 95 \mathrm{AktG}$

$\left.{ }^{2}\right)$ Johannes Semler, Corporate Governance - Beratung durch Aufsichtsratsmitglieder, NZG 2007, 882; Kalss in Doralt/Nowotny/Kalss (Hrsg), Kommentar zum Aktiengesetz (2003) § $95 \mathrm{Rz} 14$ ff; s auch Hügel, Beratung durch Aufsichtsratsmitglieder, GesRZ 1996, 215.

$\left.{ }^{3}\right)$ Die Überwachung umfasst auch zukunftsrelevante (insb strategische) Entscheidungen des Vorstands. Das ergibt sich in Österreich unter anderem aus den Zustimmungserfordernissen (§ 95 Abs $5 \mathrm{Z} 4$ AktG: wesentliche Investitionen; $Z$ 7: Aufnahme von Geschäftszweigen und Produktionsorten; $\mathrm{Z}$ 8: Festlegung allgemeiner Grundsätze der Geschäftspolitik) sowie aus den Berichtspflichten des Vorstands gegenüber dem Aufsichtsrat ( $\$ 81$ AktG). fenden Beratung zwischen Vorstand und Aufsichts$\mathrm{rat}^{4}$ ). Jedes Aufsichtsratsmitglied hat dabei seine besondere Expertise einzubringen ${ }^{5}$ ). Sind Aufsichtsratsmitglieder vom Vorstand abhängig, behindert sie das bei der Erfüllung beider Aufgaben.

In der Diskussion um die Unabhängigkeit der Aufsichtsratsmitglieder ${ }^{6}$ ) steht meist deren Unabhängigkeit vom Mehrheitsgesellschafter im Vordergrund. Hier interessiert hingegen primär das Verhältnis des Aufsichtsrats zum Vorstand $\left.{ }^{7}\right)$. Ob-

$\left.{ }^{4}\right)$ Lutter/Krieger, Rechte und Pflichten des Aufsichtsrats $^{4}$ (2002) Rz 768 ff; Kalss in Doralt/Nowotny/Kalss, AktG § $95 \mathrm{Rz} 14$; einschränkend $A$. Jäger, Die Beratung des Vorstands als Teil der Überwachungsaufgabe des Aufsichtsrats, DStR 1996, 671 ff; rechtsvergleichend (USA) und zur Kodifikation der Business Judgment Rule in Deutschland $M$. Roth, Haftung des Aufsichtsrats, GesRZ-Sonderheft Oktober 2005, 14 f.

$\left.{ }^{5}\right)$ Unterschiedliche Belastungen für einzelne Aufsichtsratsmitglieder sind daher möglich; Semler, NZG 2007, 883; Kropff in Semler/v. Schenck, Arbeitshandbuch für Aufsichtsratsmitglieder ${ }^{2}$ (2004) $\S 8 \mathrm{Rz} 114$; beide mwN.

${ }^{6}$ ) Vgl die Empfehlung der Europäischen Kommission zur Unabhängigkeit der Mitglieder von Überwachungsorganen vom 5.5.2004 sowie die Auswertung der verschiedenen Ansätze in den einzelnen Mitgliedstaaten vom 13.7.2007 (beide online verfügbar: http://ec.europa. eu/internal_market/company/independence/index_en.htm; zuletzt besucht am 8.2.2008). Rechtsvergleichend dazu Nagel, Unabhängigkeit der Kontrolle im Aufsichtsrat und Verwaltungsrat: Der Konflikt zwischen der deutschen und der angelsächsischen Konzeption, NZG 2007, 166 ff; Langenbucher, Wettbewerbsverbote, Unabhängigkeit und die Stellung des Aufsichtsratsmitglieds, ZGR 2007, 571 ff; Hopt, Aktienrecht unter amerikanischem Einfluss, FS Canaris II (2007) 116; Vetter, Aufsichtsratsvergütung und Verträge mit Aufsichtsratsmitgliedern, ZIP 2008, 5

$\left.{ }^{7}\right)$ Die Unabhängigkeit des Aufsichtsrats vom Vorstand steht allerdings häufig in einem Zusammenhang mit der 
wohl es kein allgemeines Unabhängigkeitsgebot für Aufsichtsratsmitglieder gibt ${ }^{8}$ ), steht außer Zweifel, dass sich ihre Entscheidungen am Wohl des Unternehmens orientieren müssen ${ }^{9}$ ).

Um die organisatorische Unabhängigkeit des Aufsichtsrats vom Vorstand herzustellen, ist die Hauptversammlung (und nicht der Vorstand) für die Bestellung und Abberufung der Aufsichtsratsmitglieder ${ }^{10}$ ) sowie für die Festlegung ihrer Vergütung zuständig ${ }^{11}$ ). Der Gedanke hinter dieser $\mathrm{Zu}-$ ständigkeit der Hauptversammlung ist, dass der Vorstand die Vergütung des Aufsichtsrats nicht festlegen darf und sie auch nicht beeinflussen sollte, weil damit die Überwachungsfunktion des Aufsichtsrats gefährdet würde. Nur durch einen Beschluss der Hauptversammlung kann Aufsichtsratsmitgliedern eine Sondervergütung für besonders arbeitsintensive Aufsichtsratstätigkeit gewährt werden $\left.(\S 98 \mathrm{AktG})^{12}\right)$. Hingegen konnte nach der Praxis über die Aufsichtsratstätigkeit hinausgehende Beratung mit dem Vorstand ${ }^{13}$ ) frei

Unabhängigkeit des Aufsichtsrats vom Mehrheitsgesellschafter. Besteht zwischen Vorstand und beherrschendem Gesellschafter ein enges Verhältnis, führt die Abhängigkeit der Aufsichtsratsmitglieder vom beherrschenden Gesellschafter mittelbar zu einer gewissen Abhängigkeit vom Vorstand. Dazu Peter Doralt, Die Unabhängigkeit des Vorstandes nach österreichischem und deutschem Aktienrecht - Schein und Wirklichkeit, FS Oskar Grün (2003) $31 \mathrm{ff}$.

${ }^{8}$ ) Eine besonders intensive Interessenkollision bzw Abhängigkeit ist freilich in $\S 90 \mathrm{Abs} 1 \mathrm{AktG}$ verboten (Bestellung von Vorstandsmitgliedern und Mitarbeitern zu Aufsichtsratsmitgliedern); s OGH 28.9.1960, HS 354/ 90; OGH 25.9.1997, SZ 70/189.

$\left.{ }^{9}\right)$ Vgl Schima, Organ-Interessenkonflikte und Corporate Governance, GesRZ 2003, $200 \mathrm{ff}$.

$\left.{ }^{10}\right)$ Einzige Alternative wäre die Festlegung der eigenen Vergütung durch den Aufsichtsrat selbst - damit wäre aber die Versuchung der „Selbstbedienung“ verbunden. S Godin/Wilhelmi, Aktiengesetz ${ }^{2}$ (1950) Anm II 2.; S. Wilhelmi, Sondervergütungen für Aufsichtsratsmitglieder nach dem neuen Aktiengesetz, BB 1966, 1173; Fischer, Sondervergütungen an Aufsichtsratsmitglieder, BB 1967, 861; Lutter/Kremer, Die Beratung der Gesellschaft durch Aufsichtsratsmitglieder, ZGR 1992, 92.

11) Über die Vergütung des Aufsichtsrats entscheidet die Hauptversammlung mit Beschluss oder per Satzungsbestimmung. Nebenbei sichert das eine gewisse Publizität der Bezüge. Dazu S. Wilhelmi, BB 1966, 1173; Mertens, Beratungsverträge mit Aufsichtsratsmitgliedern, FS Steindorff (1990) 174; Lutter/Kremer, ZGR 1992, $92 \mathrm{f}$. Zur abweichenden Situation bei fakultativem Aufsichtsrat und Übertragung der Vergütungskompetenz Lutter, Beraterverträge mit Aufsichtsratsmitgliedern in Gesellschaft und Konzern, FS Harm Peter Westermann (2008) 1177.

${ }^{12}$ ) In der Praxis wird die Mehrbelastung regelmäßig pauschal durch einen Aufschlag von meist $100 \%$ für den Vorsitzenden des Aufsichtsrats und $50 \%$ für seine Stellvertreter berücksichtigt, weiters häufig durch Sitzungsgelder, mit denen auch intensive Ausschusstätigkeit abgegolten werden darf. Vgl Kalss in Doralt/Nowotny/Kalss, AktG § $98 \mathrm{Rz} 19 \mathrm{f}$.

${ }^{13}$ ) Ihm obliegt die Leitung und die Vertretung der Gesellschaft nach außen, $\S \S 70 \mathrm{f} \mathrm{AktG}$. vereinbart und honoriert werden (solange es keine Sonderregel gab). Der Vorstand konnte dadurch die Loyalität eines Aufsichtsratsmitglieds belohnen oder unerwünschtes Verhalten sanktionieren $^{14}$ ). Die Einflussnahme auf einzelne oder mehrere Aufsichtsratsmitglieder war damit fast ohne Einschränkung, jedenfalls ohne Transparenz möglich.

Dem wollte der Gesetzgeber mit $\S 95$ Abs 5 Z 12 AktG begegnen. Vorbilder der mit dem GesRÄG $2005^{15}$ ) eingeführten Bestimmung waren die $\S \S 114 \mathrm{f}$ dAktG und die bisherige Regel 49 des österr Corporate Governance Kodex aus dem Jahr 2002 (CGK). § 95 Abs 5 Z 12 AktG unterscheidet sich allerdings in vieler Hinsicht von seinen Vorbildern. Während die $\S \S 114 \mathrm{f} \mathrm{dAktG}$ neben Kreditverträgen im Grunde nur Beratungsverträge erfassen, geht die österr Regelung weit darüber hinaus. Zweifelsfragen, die sich bei Regel 49 CGK aF ergaben und aufgrund der Interpretation des Arbeitskreises für Corporate Governance "lax“ gehandhabt worden waren, sind eindeutig in einem strengeren Sinn gelöst.

\section{B. Geschäfte mit Aufsichtsratsmitgliedern - wel- che Sachverhalte, welche Gefahren?}

Bei Geschäften zwischen der Gesellschaft und Aufsichtsratsmitgliedern ist zunächst zu klären, ob die zu erbringende Leistung in den Aufgabenbereich der Aufsichtsratstätigkeit fällt. Dann kann eine allfällige Sondervergütung für den besonderen Aufwand nur die Hauptversammlung, nicht der Vorstand gewähren $\left.{ }^{16}\right)$. Die zwischen einem Aufsichtsratsmitglied und dem Vorstand getroffene Vereinbarung einer gesonderten Entlohnung für eine solche Tätigkeit ist unwirksam ${ }^{17}$ ).

Im Folgenden geht es aber nur um "Geschäfte“ zwischen Aufsichtsratsmitglied und Gesellschaft über Leistungen, die nicht zu den Organpflichten gehören, also außerhalb der Aufsichtsratszuständigkeit erbracht werden ${ }^{18}$ ). Die Abgrenzung der

$\left.{ }^{14}\right)$ Grundsätzlich zur Gefahrenlage Hopt/M. Roth, AktG Großkommentar ${ }^{4}$ (2005) § 114 Rz 4 ff; s auch Haberer, Corporate Governance (2003) 192, 213. Zu meiner Analyse s B.I.

${ }^{15}$ ) BGBl I 2005/59; eine gleichlautende Bestimmung enthält § 30j Abs 5 Z 10 GmbHG.

${ }^{16}$ ) Zur Sonderfrage, ob der Aufsichtsrat nach dem dAktG 1965 (in seiner ursprünglichen Fassung) bei Vorliegen einer ermächtigenden Satzungsbestimmung einzelnen Mitgliedern eine Sondervergütung gewähren durfte, Fischer, Sondervergütungen für Aufsichtsratsmitglieder, BB 1967, 859 ff; Bernhardt, Sondervergütungen für Aufsichtsratsmitglieder, BB 1967, 865 ff; beide lehnen das überzeugend $a b$.

17) BGHZ 114, 127 = ZIP 1991, 653 ff; Brandner, Der Hausanwalt einer Aktiengesellschaft als Mitglied des Aufsichtsrats, FS Geiß (2000) 235; Hügel, GesRZ 1996, 216; Lutter/Kremer, ZGR 1992, 88; Mertens, FS Steindorff $175 \mathrm{f}$.

${ }^{18}$ ) Zur Abgrenzung Hügel, GesRZ 1996, 216 ff; Lutter/ Kremer, ZGR 1992, 93 ff; Kropff in Semler/v. Schenck, Arbeitshandbuch ${ }^{2} \S 8 \mathrm{Rz} 112 \mathrm{ff} ;$ Hopt/M. Roth, $\mathrm{AktG}^{4}$ $\S 114 \mathrm{Rz} 16$ ff. Geßler in Geßler/Hefermehl/Eckhardt/ 
Aufsichtsratstätigkeit von sonstigen Beratungsleistungen hat $\mathrm{zu}$ ausführlichen Diskussionen geführt. Richtigerweise kommt es dabei weder auf den Umfang noch auf die Intensität der Beratungsleistung ${ }^{19}$ ), sondern den Inhalt $\mathrm{an}^{20}$ ).

\section{Grundfall: Beratungsvertrag}

Verboten ist die entgeltliche Beratung durch Aufsichtsratsmitglieder nicht, und das ist gut so. Denn in Ausnahmefällen mag es Gründe geben, ein Aufsichtsratsmitglied zur Beratung der Gesellschaft heranzuziehen $-\mathrm{zB}$ wenn gerade dessen ganz außergewöhnliche fachliche Qualifikation benötigt wird. Weil die entgeltliche Beratung der Gesellschaft durch Aufsichtsräte aber Interessenkonflikte und Abhängigkeiten schafft, sollte sie nicht den Regelfall bilden. Jedenfalls gilt das, soweit die Beratung gegenüber dem Vorstand zu erbringen ist. Erst recht sollte die Beratung durch ein Aufsichtsratsmitglied kritisch überdacht werden, wenn vergleichbare Sachkunde und Effizienz von anderen Personen als dem Aufsichtsratsmitglied erhältlich sind ${ }^{21}$ ). Um folgende Gefahren geht es bei der Beratung der Gesellschaft durch ein Aufsichtsratsmitglied ${ }^{22}$ ):

\section{Vorteilszuwendung und Beeinflussung der kol- lektiven Willensbildung im Aufsichtsrat}

Konkrete „Anordnungen“ oder „Weisungen“ sind in einem Abhängigkeitsverhältnis oft nicht erforderlich, um ein gewünschtes Verhalten herbeizuführen. Vorauseilender Gehorsam oder ein diskreter Hinweis genügen meist.

Kropff (Hrsg), Aktiengesetz (1973, 1974) § $114 \mathrm{Rz} 5 \mathrm{ff}$ Mertens in Kölner Kommentar zum Aktiengesetz $(\mathrm{KK})^{2}$ (1996) § $114 \mathrm{Rz} 4 \mathrm{f}$.

$\left.{ }^{19}\right)$ Kropff in Semler/v. Schenck, Arbeitshandbuch ${ }^{2} \S 8$ $\mathrm{Rz} 114$.

${ }^{20}$ ) Argumente dafür bei Lutter/Kremer, ZGR 1992 93 ff; Hopt/M. Roth, AktG ${ }^{4} \S 114$ Rz 17; Kropff in Semler/v. Schenck, Arbeitshandbuch ${ }^{2} \S 8 \mathrm{Rz} 116$; Benecke, Beratungsvereinbarungen mit Aufsichtsratsmitgliedern, WM 2007, 717 ff; vgl auch BGHZ 126, 344; Lutter, FS Harm Peter Westermann 1172 ff. Für diese Abgrenzung spricht unter anderem, dass der Umfang der Beratungsund Kontrollpflichten des Aufsichtsrats (abgesehen von Mindestanforderungen) nicht im Gesetz festgelegt ist (Lutter/Kremer, ZGR 1992, 94) und teils vom Bedarf abhängen muss. So wird zB in schwierigen Geschäftssituationen, wie in der Krise, allgemein eine Intensivierung der Aufsichtsratstätigkeit verlangt (Johannes Semler in Semler/v. Schenck, Arbeitshandbuch ${ }^{2} \& 1 \mathrm{Rz} 80 ; \mathrm{s}$ auch OLG Stuttgart 15.3.2006, ZIP 2006, 756 f). Aus der Praxis stammende Beispiele für Leistungsverpflichtungen bei Verträgen mit Aufsichtsratsmitgliedern finden sich bei Vetter, ZIP 2008, 6.

$\left.{ }^{21}\right)$ Nachdrücklich Kropff in Semler/v. Schenck, Arbeitshandbuch $^{2} \& 8 \mathrm{Rz} 126$. Ebenso Semler, NZG 2007, 887, der bei der Mitgliedschaft im Aufsichtsrat rät, entweder darauf oder auf die Beratung der Gesellschaft zu verzichten.

${ }^{22}$ ) Vgl Semler, NZG 2007, 886; rechtsvergleichend s Faulhaber, Beratertätigkeit mit Unternehmenskontrolleuren im deutschen und englischen Recht (2004) $30 \mathrm{ff}$.
Großzügig entlohnte Beratungsleistungen können ein Aufsichtsratsmitglied freundlich stimmen. Aus Sicht des Vorstands ist das kein Nachteil ${ }^{23}$ ), eher ein angenehmer Nebeneffekt. Vielleicht kann so die Unterstützung des Mitglieds gewonnen werden (eventuell des einzigen mit fachspezifischem Wissen). Zusatzberatung steht in einem Spannungsverhältnis zur Kontrollfunktion des Aufsichtsrats, und die allzu leicht entstehende Abhängigkeit ist problematisch. In der Praxis sind großzügig entlohnte Beratungsverträge mit Aufsichtsratsmitgliedern häufig ${ }^{24}$ ). Das schafft finanzielle Anreize und Eigeninteressen ${ }^{25}$ ). Die allgemeine Eignung finanzieller Anreize zur Verhaltenssteuerung steht außer Frage.

Erleichtert wird die Beeinflussung des Aufsichtsrats als Kollegialorgan, wenn die anderen Aufsichtsratsmitglieder über die finanziellen Anreize des einzelnen Mitglieds nicht informiert sind $\left.{ }^{26}\right)$. Im Interesse der unverfälschten kollektiven Willensbildung ist die Transparenz gegenüber den anderen Aufsichtsratsmitgliedern zu sichern $^{27}$ ). Neben der Gefahr des Unabhängigkeitsverlusts des einzelnen Aufsichtsratsmitglieds geht es um den Schutz des Aufsichtsrats insgesamt vor unsachlicher und unbewusster Beeinflus$\left.\operatorname{sung}^{28}\right)$.

\section{Verlust der Distanz zum Vorstand}

Das Aufsichtsratsmitglied muss das Wohlwollen und Vertrauen des Vorstands genießen, um überhaupt $\mathrm{zu}$ Beratungsleistungen herangezogen $\mathrm{zu}$ werden. Um diese Einkunftsquelle nicht zu gefährden, gilt es, ein optimales Verhältnis zu bewahren. Gutes Einvernehmen ist nützlich, große Nähe hingegen problematisch. Mangelnde Distanz zwischen Vorstand und Aufsichtsrat birgt erhebliches Potential für Interessenkonflikte, etwa bei der Verlängerung (oder Beendigung) eines Vorstandsvertrags, dessen genauer Ausgestaltung oder der Ge-

23) Peltzer, Beratungsverträge der Gesellschaft mit Aufsichtsratsmitgliedern: Ist das gute Corporate Governance?, ZIP 2007, 308.

${ }^{24)}$ Vetter, Beratungsverträge mit Aufsichtsratsmitgliedern, AG 2006, 173; R. Werner, Die Beratung der Aktiengesellschaft durch Mitglieder ihres Aufsichtsrats, DB 2006, 935; Lutter, FS Harm Peter Westermann 1172.

$\left.{ }^{25}\right)$ Laut Vetter, AG 2006, 173 dienen in der Praxis solche Verträge häufig der Gewährung einer zusätzlichen Vergütung für Aufsichtsratsmitglieder; s auch Vetter, ZIP 2008, 6 (insb die Beispiele aus der Rsp in FN 66).

${ }^{26}$ ) Diese Gefahr wird vielfach übersehen, auch wenn sie praktisch im mehrpersonalen Diskussionsprozess und in der kollektiven Willensbildung einen erheblichen Einfluss haben dürfte.

${ }^{27)}$ Potyka, Das Gesellschaftsrechtsänderungsgesetz 2005, ÖJZ 2006, 195.

${ }^{28}$ ) Peltzer, ZIP 2007, 309; s dort insb FN 52 mit Verweis auf Sihler, WPg Sonderheft Dezember 2001, $11 \mathrm{ff}$ und die Hinweise $\mathrm{zu}$ den praktischen Abläufen der kollektiven Entscheidungsfindung im Aufsichtsrat sowie zur faktisch äußerst beschränkten Möglichkeit, innerhalb einer Sitzung sich abzeichnende Entscheidungstendenzen noch einmal umzukehren. 
währung einer Prämie ${ }^{29}$ ). Bei sachlichen Differenzen, die uU schnelles und entschiedenes Handeln verlangen, kann ein Naheverhältnis Zurückhaltung oder Verzögerungen bewirken. Bereits die bloße Möglichkeit zur Schaffung konkreter, finanzieller Anreize baut hier latente Interessenkonflikte auf.

\section{Verlust der unbefangenen Kontrollfunktion für die konkrete Fragestellung}

Berät ein Aufsichtsratsmitglied den Vorstand, kann es dieses Thema nicht nachträglich unvoreingenommen in der Funktion des Aufsichtsratsmitglieds kontrollieren. Besonders die Beratung des Vorstands im Zusammenhang mit grundlegenden und strategischen Entscheidungen ist problematisch, weil damit für die Aufsichtsratstätigkeit der Blick von außen, also von außerhalb der Geschäftsführung, verloren geht.

Die Funktion des Aufsichtsrats wird erleichtert, wenn die Beratung über Details einer Vertragsgestaltung von der strategischen Beratung und Kontrolle personell getrennt ist. Nichts spricht aber dagegen, einen Berater, der die Vertragsgestaltung für die Gesellschaft durchführt, dem Aufsichtsrat berichten zu lassen und ihn vor der Beschlussfassung anzuhören. Die gleichzeitige Mitgliedschaft des Beraters im Aufsichtsrat ist dafür nicht erforderlich.

\section{Nicht fremdübliche Bedingungen}

Schließlich ist denkbar, dass das Entgelt für die Leistung des Aufsichtsratsmitglieds besonders großzügig ist, also zu nicht fremdüblichen Bedingungen kontrahiert wird. Das verschärft die Gefahrenlage (und rückt die Angelegenheit in die Nähe der Untreue).

\section{Regelungsproblem bei Beratungsleistungen (Geschäften) ,mit Unternehmen, an denen das Aufsichtsratsmitglied ein erhebliches wirt- schaftliches Interesse hat"}

Ähnliche Risiken entstehen, wenn nicht das Aufsichtsratsmitglied selbst, sondern ein ihm nahestehendes Unternehmen die Leistung erbringt: Die Gesellschaft wird durch die Anwaltskanzlei oder Wirtschaftsprüfungsgesellschaft, der das Aufsichtsratsmitglied angehört, beraten. Ob das Aufsichtsratsmitglied an der Erbringung der Leistung beteiligt ist oder bloß seine Kanzleikollegen, macht nur einen geringen Unterschied. In beiden Fällen entstehen direkte oder indirekte finanzielle Vorteile durch das Mandat. Beratung durch Aufsichtsratsmitglieder oder ihnen nahestehende Gesellschaften schafft ein weitgehend gleichartiges Regelungsproblem. Die umfassende Einbeziehung

${ }^{29}$ ) Darüber wurde im Herbst 2005 bei der Verlängerung des Generaldirektors der Verbund AG (Österreichische Elektrizitätswirtschafts-AG) medial berichtet - im konkreten Fall kam es (trotz vermeintlicher oder tatsächlicher Anreize) nicht zur Wiederbestellung. von Verträgen mit Unternehmen, an denen das Aufsichtsratsmitglied ein erhebliches wirtschaftliches Interesse hat, erschwert auch Umgehungen ${ }^{30}$ ). In Deutschland, wo eine gesetzliche Regel fehlt, wird darin eine Lücke gesehen, die von der $\operatorname{Rsp}^{31}$ ) mit überwiegender Zustimmung der Lehre durch analoge Anwendung geschlossen wird ${ }^{32}$ ). Möglicherweise besteht bei der Beratung durch ein Unternehmen, an dem das Aufsichtsratsmitglied ein erhebliches wirtschaftliches Interesse hat, eine geringere Befangenheit, wenn das Aufsichtsratsmitglied nicht persönlich berät. Bei der umfassenden österr Einbeziehung aller Geschäfte (nicht nur Beratungen) ist diese Nuance aber nicht entscheidend.

\section{Vorbilder für $\S 95$ Abs 5 Z 12 AktG (und $\S 30 j$ Abs 5 Z 10 GmbHG)}

Orientiert hat sich der Gesetzgebungsprozess an $\S 114$ dAktG und Regel 49 CGK $\left.(\mathrm{aF})^{33}\right)$.

\section{I. $§ 114$ dAktG}

Der deutsche Gesetzgeber hat sich dem hier aufgegriffenen Problem bereits 1965 in $\S 114$ dAktG zugewandt ${ }^{34}$ ):

"Verträge mit Aufsichtsratsmitgliedern

(1) Verpflichtet sich ein Aufsichtsratsmitglied außerhalb seiner Tätigkeit im Aufsichtsrat durch einen Dienstvertrag, durch den ein Arbeitsverhältnis nicht begründet wird, oder durch einen Werkvertrag gegenüber der Gesellschaft zu einer Tätigkeit höherer Art, so hängt die Wirksamkeit des Vertrags von der Zustimmung des Aufsichtsrats ab.

(2) Gewährt die Gesellschaft auf Grund eines solchen Vertrags dem Aufsichtsratsmitglied eine Vergütung, ohne dass der Aufsichtsrat dem Vertrag zugestimmt hat, so hat das Aufsichtsratsmitglied die Vergütung zurückzugewähren, es sei denn, dass der Aufsichtsrat den Vertrag genehmigt. Ein Anspruch des Aufsichtsratsmitglieds gegen die Gesellschaft auf Herausgabe der durch die geleistete Tätigkeit erlangten Bereicherung bleibt unberührt; der Anspruch kann jedoch nicht gegen den Rückgewähranspruch aufgerechnet werden."

Beratungstätigkeiten durch Aufsichtsratsmitglieder waren in Deutschland auch vor 1965 weit verbreitet und meist großzügig entlohnt ${ }^{35}$ ). Mit

${ }^{30}$ ) Vgl Schauer, GesRÄG 2005: Verbesserungen bei der Corporate Governance - Rückschritte bei der Abschlussprüfung, Aufsichtsrat aktuell 2006, 5; Kalss, Organgeschäfte mit Unternehmen des Aufsichtsratsmitglieds, Aufsichtsrat aktuell 2006, 7; dieselbe, Die Zustimmungspflicht des Aufsichtsrats zu Verträgen mit Unternehmen seiner Mitglieder, SWK 2006, 420.

$\left.{ }^{31}\right) \mathrm{S}$ etwa BGH 1.7.2006, WM 2006, 1581; BGH 20.11.2006, ZIP 2007, 22; LG Stuttgart 27.5.1998, BB 1998, $1549 \mathrm{ff}$.

${ }^{32}$ ) Aktueller Überblick bei Drygala in K. Schmidt/ Lutter (Hrsg), Aktiengesetz Kommentar (2008) § 113 Rz 17 f; Lutter, FS Harm Peter Westermann 1172 ff. Näheres unter C.I.

${ }^{33}$ ) Erl RV 927 BlgNR 22. GP 8.

$\left.{ }^{34}\right) \mathrm{Vgl}$ Kropff, Aktiengesetz (1965) 158 f; Geßler in Geßler/Hefermehl/Eckhardt/Kropff, AktG § $114 \mathrm{Rz} 1$.

${ }^{35}$ ) Geßler in Geßler/Hefermehl/Eckhardt/Kropff, AktG § 114 Rz 1. Zur Entschärfung dieses Problems hatte 
dem dAktG 1965 hat der deutsche Gesetzgeber durch Zuweisung der Entscheidungskompetenz an den Aufsichtsrat ${ }^{36}$ ) auf Information und Kontrolle der übrigen Aufsichtsratsmitglieder gesetzt $\left.{ }^{37}\right)$. Eine reine Transparenzvorschrift ist $\S 114$ dAktG aber nicht: Der Aufsichtsrat kann die $\mathrm{Zu}-$ stimmung verweigern.

Mehrfach ist der Anwendungsbereich von $\S 114$ dAktG durch Abs 1 beschränkt ${ }^{38}$ ). Grundsätzlich werden andere als die genannten Verträge nicht erfasst $^{39}$ ). Nur für Kreditverträge gibt es in $\S 115$ dAktG eine Sonderregelung. Unterschiedliche Standpunkte gibt es zur Analogiefähigkeit der $\S \S 114 \mathrm{f} \mathrm{dAktG}^{40}$ ). Das betrifft die Ausdehnung auf andere Geschäftstypen als freie Dienstverträge, auf Konzernverträge ${ }^{41}$ ), auf Geschäfte des Aufsichtsratsmitglieds mit Tochtergesellschaften ${ }^{42}$ ) sowie auf Geschäfte zwischen der Gesellschaft und Unternehmen, an denen das Aufsichtsratsmitglied maßgeblich beteiligt ist ${ }^{43}$ ) oder die diesem sonst na-

deshalb das deutsche Schrifttum vor der Einführung des $\S 114 \mathrm{dAktG}$ vereinzelt angenommen, dass alle Geschäfte der Gesellschaft mit Aufsichtsratsmitgliedern der $\mathrm{Zu}$ stimmung der Hauptversammlung bedürfen; Godin/Wilhelmi, Aktiengesetz ${ }^{3}$ (1967) § 114 Anm I. Die Problematik war also bereits vor der Einführung des $§ 114 \mathrm{dAktG}$ erkannt worden. Trotz der bis 2005 für Österreich vergleichbaren Rechtslage wurde eine Kompetenz der Hauptversammlung soweit ersichtlich nie angenommen.

${ }^{36}$ ) An das schwerfällige Instrument der Zustimmung durch die Hauptversammlung (meist nur jährlich möglich) wollte man die Zulässigkeit schon aus Praktikabilitätsgründen nicht binden. Aus guten Gründen wurde auch bei der Beratung des KonTraG (Gesetz zur Kontrolle und Transparenz im Unternehmensbereich, dBGBl I 1998, 786) der Vorschlag verworfen, statt der Zustimmung des Aufsichtsrats jene der Hauptversammlung vorzuschreiben. S Hopt/M. Roth, $\mathrm{AktG}^{4} \S 114 \mathrm{Rz} 3 \mathrm{mwN}$; Semler in Münchener Kommentar zum Aktiengesetz ${ }^{2}$ (2004) § $114 \mathrm{Rz} 14$.

${ }^{37}$ ) Vgl Semler in MünchKomm $\mathrm{AktG}^{2} \S 114 \mathrm{Rz} 1 \mathrm{ff}$; Hopt/M. Roth, $\mathrm{AktG}^{4} \S 114 \mathrm{Rz} 5$.

${ }^{38}$ ) Nur Dienstverträge, durch die kein Arbeitsverhältnis begründet wird, und Werkverträge; vgl zB Beater, Beratungsverträge für Aufsichtsratsmitglieder $(\S \S 113,114$ AktG), ZHR 157 (1993) 427; vgl Hüffer, Aktiengesetz (2006) § $114 \mathrm{Rz} 3$; Hopt/M. Roth, $\mathrm{AktG}^{4} \S 114 \mathrm{Rz} 8$.

${ }^{39}$ ) Geßler in Geßler/Hefermehl/Eckhardt/Kropff, AktG $\S 114 \mathrm{Rz}$ 5; Beater, ZHR 157 (1993) 427 f; Hopt/ M. Roth, $\mathrm{AktG}^{4} \S 114 \mathrm{Rz} 12$

${ }^{40}$ ) Überblick bei Drygala in K. Schmidt/Lutter, AktG $\S 113 \mathrm{Rz} 17 \mathrm{f}$.

$\left.{ }^{41}\right)$ Dazu im Einzelnen nun Lutter, FS Harm Peter Westermann $1172 \mathrm{ff}$.

${ }^{42}$ ) S FN 31 und 32; gegen die Anwendung auf Verträge mit Tochtergesellschaften Mertens, FS Steindorff 186: Der Gesetzgeber habe in $\S 115$ dAktG eine Regelung für Kreditgeschäfte auch im Zusammenhang mit Tochtergesellschaften getroffen; damit scheide im Umkehrschluss die Anwendung auf Tochtergesellschaften bei $\S 114$ dAktG aus. AA Lutter/Kremer, ZGR 1992, 105 f, die den Umkehrschluss nicht für zwingend halten und auf den Telos der Norm - Vermeidung unsachlicher Einflüsse - abstellen und die Analogie bejahen; deutlich auch Lutter, FS Harm Peter Westermann $1178 \mathrm{f}$.

${ }^{43}$ ) Die analoge Anwendung von $\S 114$ dAktG auf diese Verträge wird überwiegend anerkannt, jedoch mit unter- hestehen ${ }^{44}$ ). Für Geschäfte, die vor der Bestellung zum Aufsichtsratsmitglied geschlossen wurden, ist die Anwendung von § $114 \mathrm{dAktG}$ angenommen worden. Sogar für die Übernahme der Aufsichtsratstätigkeit in einer Tochtergesellschaft durch ein Mitglied des Aufsichtsrats der Muttergesellschaft wurde die Zustimmungspflicht erwogen ${ }^{45}$ ).

\section{1. „Bringschuld“ sowohl des Vorstands als auch des betroffenen Aufsichtsratsmitglieds}

Die Pflicht und das Recht zur Befassung des Aufsichtsrats trifft nach ganz herrschender und

schiedlicher Reichweite. Für eine Anwendung unabhängig vom Ausmaß der Beteiligung des Aufsichtsratsmitglieds an der Sozietät bzw dem Unternehmen, mit dem kontrahiert wird: Oppenhoff, Zum Umkreis der von $\S 114$ AktG Betroffenen, FS Barz (1974) 283 ff; Rellermeyer, Der Aufsichtsrat, ZGR 1993, 87 ff; Hellwig, Beratungsverträge des Abschlussprüfers - Genehmigungspflicht analog $\S 114$ AktG und Publizitätspflicht analog $\S 125$ Abs 1 Satz 3 AktG, ZIP 1999, 2127. Hingegen für die Anwendung auf Verträge mit vom Aufsichtsratsmitglied abhängigen Unternehmen Lutter/Kremer, ZGR 1992, 106. Für die Anwendung, soweit das Aufsichtsratsmitglied selbst im Rahmen des Vertrags zwischen der Gesellschaft und seiner Sozietät (bzw seinem Unternehmen) an der Leistungserbringung mitwirken soll: Mertens, KK $\mathrm{AktG}^{2} \S 114 \mathrm{Rz} 7$. Grundsätzlich für die analoge Anwendung auch Johannes Semler in MünchKomm AktG ${ }^{2} \S 114$ Rz 42 ff; Vetter, AG 2006, 178. Gegen die Anwendung auf Verträge mit der Sozietät des Aufsichtsratsmitglieds Heussen, Interessenkonflikte zwischen Amt und Mandat bei Aufsichtsräten, NJW 2001, 709; Wissmann/Ost, Im Blickpunkt: Der Beratungsvertrag mit der Sozietät eines Aufsichtsratsmitglieds, BB 1998, 1960. Lutter/Drygala, Die besondere sachverständige Beratung des Aufsichtsrats durch seine Mitglieder, FS Ulmer (2003) 383 ff kam es bei Beauftragung der Anwaltssozietät auf den finanziellen Vorteil des Aufsichtsratsmitglieds für die Anwendung von $\S 114 \mathrm{dAktG}$ an, auch wenn dieser nur mittelbar war. Ebenso nun Lutter, FS Harm Peter Westermann 1180 und BGH ZIP 2007, 22 (dazu unten bei D.III.). Einschränkend aber noch Lutter/Drygala bei Beauftragung einer Steuerberatungs- oder Wirtschaftsprüfungsgesellschaft in Form einer GmbH oder AG: $\$ 114$ dAktG sei nicht anwendbar - auch nicht aus teleologischen Gründen: „[. . .] Interessenkonflikte sind [. . .] in Aufsichtsräten keine Besonderheit und [...] hier nicht stärker als bei einem Aufsichtsratsmitglied, dessen Gesellschaft Lieferant, Abnehmer oder sonst Gläubiger des Unternehmens ist [...]." Das überrascht aus österr Sicht, erklärt sich aber schlicht mit der unterschiedlichen Gesetzeslage und der relativen Schwäche von $\S 114 \mathrm{dAktG}$, der viele dem Beratungsverhältnis ähnliche Fälle von Interessenkonflikten nicht erfasst. Für solche bedarf es in Deutschland weder der Zustimmung noch der Information der anderen Aufsichtsratsmitglieder. Wenigstens die Information über diese Verträge mit einem Mitglied entspricht aber in der Praxis dem ,guten Stil, [soweit sie] für das Mitglied erheblich sind und außerhalb des Tagesgeschäfts liegen" (Kropff in Semler/v. Schenck, Arbeitshandbuch ${ }^{2}$ \& $\left.8 \mathrm{Rz} 110\right)$.

$\left.{ }^{44}\right)$ Der BGH hat $\S 114$ dAktG auf solche Fälle immer wieder angewandt; zB BGH 3.7.2006, ZIP 2006, 1529; BGH 2.4.2007, DB 2007, 1296; kritisch Happ, Anwaltlicher Beratungsvertrag und Aufsichtsratsmandat, FS Priester (2007) $175 \mathrm{ff}$.

${ }^{45}$ ) Lutter/Kremer, ZGR 1992, 107. 
zutreffender Auffassung sowohl den Vorstand als auch das betroffene Aufsichtsratsmitglied ${ }^{46}$ ).

\section{Erfordernis konkreter Information über den In- halt des Geschäfts}

Für die Zustimmung (oder Ablehnung) des Aufsichtsrats bedarf es vorher entsprechender Information. Der BGH stellt - ganz zu Recht und mit Zustimmung der Lehre - hohe Anforderungen:

Dem Aufsichtsrat sind die konkreten Leistungsverpflichtungen mitzuteilen ${ }^{47}$ ). Inhalt und Umfang der Leistung sind genau darzulegen ${ }^{48}$ ). Damit soll der Aufsichtsrat zunächst feststellen, ob die Tätigkeit bereits im Rahmen der Aufsichtsratstätigkeit geschuldet wird. Dann käme eine Zustimmung nicht in Frage, und die Vereinbarung wäre ohnehin nichtig ${ }^{49}$ ). Die Anforderungen an die konkrete Beschreibung der Leistungsverpflichtung sind hoch. Die beispielhafte Umschreibung reichte dem BGH nicht aus ${ }^{50}$ ). Auch das Schrifttum stellt hohe Anforderungen („umfassendes Transparenzgebot $\left.{ }^{(51)}\right)$ ).

Ebenso muss das Entgelt offengelegt werden, damit sich der Aufsichtsrat ein Urteil über die Angemessenheit der Vergütung bilden kann ${ }^{52}$ ). Steht das Entgelt vorab nicht fest, ist die Art der Berechnung so genau wie möglich festzulegen und mitzuteilen. Dabei sind Obergrenzen zu bestimmen ${ }^{53}$ ). Die Mitteilung, es werde nach ,üblichen Stundensätzen" verrechnet ${ }^{54}$ ), genügt der Rsp ebenso wenig wie der Verweis auf standesrechtliche oder gesetzliche Tarife.

Die Beurteilung des Vertrags durch den Aufsichtsrat ohne die genannten Informationen kann den gesetzlichen Zweck nicht erfüllen, da es gerade um die Offenlegung aller Inhalte geht, die

${ }^{46}$ ) Vgl Semler in MünchKomm $\mathrm{AktG}^{2} \S 114 \mathrm{Rz} 54$; Hüffer, $\mathrm{AktG}^{7} \S 114 \mathrm{Rz} 6$; Kropff in Semler/v. Schenck, Arbeitshandbuch ${ }^{2} \S 8 \mathrm{Rz} 123$; Vetter, AG 2006, 178; jeweils $\mathrm{mwN}$.

$\left.{ }^{47}\right)$ Hopt/M. Roth, $\mathrm{AktG}^{4} \S 114 \mathrm{Rz} 52$ f; Kropff in Semler/v. Schenck, Arbeitshandbuch ${ }^{2} \S 8 \mathrm{Rz} 116$; Mertens, KK $\mathrm{AktG}^{2} \S 114 \mathrm{Rz} 6$; Beispiele bei Vetter, ZIP 2008, 7.

$\left.{ }^{48}\right)$ BGHZ 126, $344 \mathrm{f}$.

$\left.{ }^{49}\right)$ Betrifft dies nur Teile der Vereinbarung, die sich aber nicht klar abgrenzen lassen, ist im Zweifel die gesamte Vereinbarung nichtig: Hopt/M. Roth, $\mathrm{AktG}^{4} \S 114$ Rz 22 ff; Lutter, FS Harm Peter Westermann 1176; Lutter/Kremer, ZGR 1992, 96; Vetter, ZIP 2008, 7; aA Beater, ZHR 157 (1993) 434.

${ }^{50}$ ) Vgl BGH 20.11.2006, ZIP 2007, 22; Besprechungsaufsatz von Peltzer, ZIP 2007, 305 ff; vgl Benecke, WM 2007, 717 ff; G. Weiss, Beratungsverträge mit Aufsichtsrats- und Beiratsmitgliedern in der Aktiengesellschaft und der Gesellschaft mit beschränkter Haftung, BB 2007, $1853 \mathrm{ff}$.

${ }^{51}$ ) Kropff in Semler/v. Schenck, Arbeitshandbuch ${ }^{2} \S 8$ $\mathrm{Rz} 124$.

${ }^{52}$ ) BGHZ 126, 345. Vgl Semler in MünchKomm AktG ${ }^{2}$ $\S 114 \mathrm{Rz} 78$.

$\left.{ }^{53}\right)$ Hopt/M. Roth, $\mathrm{AktG}^{4} \S 114 \mathrm{Rz} 54$.

${ }^{54)}$ LG Stuttgart ZIP 1998, 1279. Soweit zu erfahren ist, hält das die österr Praxis für ausreichend $(\mathrm{mE} \mathrm{zu}$ Unrecht). eine Gefährdung der Unabhängigkeit bewirken. Ohne hinreichende Information ist der Vertrag mit dem Aufsichtsratsmitglied trotz Zustimmungsbeschluss des Aufsichtsrats unwirksam ${ }^{55}$ ).

\section{Beschlusserfordernis und nachträgliche Be- schlüsse}

Die bloße Kenntnisnahme genügt nicht - § 114 dAktG verlangt die „Zustimmung“. Der Aufsichtsrat entscheidet allgemein mit Beschluss - eine konkludente Beschlussfassung sieht das Gesetz nicht vor ${ }^{56}$ ); es bedarf eines formellen Beschlus$\left.\operatorname{ses}^{57}\right)$.

Nachträgliche Beschlüsse sind nach überwiegender Ansicht wirksam. Dafür spricht, dass die Kontrolle durch die übrigen Aufsichtsratsmitglieder besser nachträglich als gar nicht erfolgen soll ${ }^{58}$ ) und dabei die Zustimmung immer noch verweigert werden $\mathrm{kann}^{59}$ ). Je länger die Vereinbarung zurückliegt, umso problematischer wird aber sein, dass der Aufsichtsrat in der Zwischenzeit nicht informiert war $^{60}$ ).

\section{Zustimmung nur bei Verträgen, die im Interesse der Gesellschaft liegen}

Der Aufsichtsrat muss prüfen, ob der zu genehmigende Vertrag mit dem Aufsichtsratsmitglied im Interesse der Gesellschaft ist ${ }^{61}$ ). Der Beschluss hat sich daran $\mathrm{zu}$ orientieren (die Zustimmung $\mathrm{zu}$ einem Vertrag, der nicht im Interesse der Gesellschaft liegt, kann zur Haftung der Aufsichtsratsmitglieder führen).

\section{Stimmverbot und Folgen für die Beschlussfä- higkeit des Aufsichtsrats}

Da ein Aufsichtsratsbeschluss erforderlich ist, stellt sich die Frage der Stimmberechtigung des betroffenen Mitglieds. Mit dem Telos der Norm (unabhängige Kontrolle des Vertragsinhalts und Beschlussfassung über dessen Zulassung) ist dessen Mitstimmen nicht vereinbar ${ }^{62}$ ).

$\left.{ }^{55}\right)$ Hopt/M. Roth, $\mathrm{AktG}^{4}$ \& $114 \mathrm{Rz} 25$; Lutter/Kremer, ZGR 1992, 96; OLG Frankfurt 21.9.2005, AG 2005, 925 ff; Vetter, AG 2006, 178; Hüffer, $\mathrm{AktG}^{7} \S 114 \mathrm{Rz} 6$.

$\left.{ }^{56}\right) \S 108$ dAktG; Hüffer, $\mathrm{AktG}^{7} \S 108 \mathrm{Rz} 4$; zu Einzelfragen Zöllner, Beschluß, Beschlußergebnis und Beschlußergebnisfeststellung, FS Lutter (2000) $821 \mathrm{ff}$.

$\left.{ }^{57}\right)$ Vetter, AG 2006, 178.

${ }^{58}$ ) Lutter/Drygala, FS Ulmer 396; Kremer in Ringleb/ Kremer/Lutter/v. Werder (Hrsg), Kommentar zum Deutschen Corporate Governance Kodex ${ }^{2}$ (2005) Rz 1125; Vetter, AG 2006, 178; Grunewald, Kombination von Beratungsverträgen und Aufsichtsratsmandaten, AnwBl 2007, 568; Benecke, WM 2007, 719; Happ, FS Priester 197; Vetter, ZIP 2008, 8.

${ }^{59)}$ M. Roth, GesRZ-Sonderheft Oktober 2005, $17 \mathrm{f}$.

${ }^{60}$ Vetter, ZIP 2008, 8 mit strengen Anforderungen.

$\left.{ }^{61}\right)$ Kropff in Semler/v. Schenck, Arbeitshandbuch ${ }^{2} \S 8$ $\mathrm{Rz} 126$.

${ }^{62}$ ) S BGH 2.4.2007, DB 2007, 1296; OLG Frankfurt 21.9.2005, AG 2005, 925 ff; Hopt/M. Roth, $\mathrm{AktG}^{4} \S 114$ $\mathrm{Rz} 47$; Semler in MünchKomm $\mathrm{AktG}^{2} \S 114 \mathrm{Rz} 79$; denselben in Semler/v. Schenck, Arbeitshandbuch ${ }^{2} \S 1 \mathrm{Rz} 223$; 
Aus dem Stimmverbot kann sich in kleineren Aufsichtsräten eine Sonderproblematik ergeben: Bei nur drei Aufsichtsratsmitgliedern stellt sich die Frage, ob das Stimmverbot die Beschlussunfähigkeit auslöst (§ 108 Abs 2 S 2 dAktG: „In jedem Fall müssen mindestens drei Mitglieder an der Beschlussfassung teilnehmen"). Nach Ansicht des BGH bleibt der Aufsichtsrat beschlussfähig, obwohl sich das betroffene Aufsichtsratsmitglied der Stimme enthalten muss ${ }^{63}$ ).

\section{Unwirksamkeit ohne Zustimmung}

$\S 114$ Abs 1 dAktG beschränkt die Vertretungsmacht des Vorstands für die Geschäfte mit den Aufsichtsratsmitgliedern: Wirksam sind die Vereinbarungen nur, wenn der Aufsichtsrat zustimmt. Wird die Zustimmung nicht eingeholt, ist das Geschäft nach $\S 114$ Abs 2 dAktG schwebend unwirksam; wird sie verweigert, besteht mangels Vertrags kein Entgeltanspruch des Aufsichtsratsmitglieds ${ }^{64}$ ); empfangene Zahlungen sind zurückzuerstatten. Allfällige bereicherungsrechtliche Ansprüche gegen die Gesellschaft bleiben davon unberührt (§ 114 Abs 2 dAktG).

\section{Aufrechnungsverbot - Konkursrisiko}

Ausgeschlossen ist die Aufrechnung des zurückzuzahlenden Honorars mit allfälligen Bereicherungsansprüchen. Im Konkurs der Gesellschaft muss das Aufsichtsratsmitglied (oder die ihm nahestehende Gesellschaft, an die das Honorar bezahlt wurde ${ }^{65}$ )) die erhaltenen Honorare zurückzahlen - allfällige Bereicherungsansprüche ${ }^{66}$ ) gegen die Gesellschaft sind dann meist uneinbringlich ${ }^{67}$ ).

Mertens, KK AktG ${ }^{2} \S 114 \mathrm{Rz} 12 ;$ Lutter/Krieger, Rechte und Pflichten des Aufsichtsrats ${ }^{4} \mathrm{Rz}$ 439; Kremer in Ringleb/Kremer/Lutter/v. Werder, Corporate Governance ${ }^{2}$ Rz 1126; nach Lutter, FS Harm Peter Westermann 1172 FN 7 muss nicht nur das Stimmrecht entfallen, sondern auch die Teilnahme an der Beratung.

$\left.{ }^{63}\right) \mathrm{BGH}$ 2.4.2007, DB 2007, 1296; anders noch OLG Frankfurt 21.9.2005, AG 2005, 925; zur E des OLG Frankfurt kritisch Bosse, Rechtliche Anforderungen an Verträge mit Aufsichtsratsmitgliedern und die Zustimmung nach $\S 114$ AktG, NZG 2007, 175; R. Werner, DB 2006, 937 f ging von einer ex-lege-Beschlussunfähigkeit des Aufsichtsrats aus. Mit der in der Sache richtigen Entscheidung des $\mathrm{BGH}$ ist allerdings schwer vereinbar, dass das Aufsichtsratsmitglied auch von der Beratung ausgeschlossen ist (Lutter, FS Harm Peter Westermann 1172 FN 7); das deutet allerdings bloß darauf hin, dass die Entscheidung des BGH besser mit einer Reduktion von $\S 108$ Abs 2 S 2 dAktG begründet worden wäre.

$\left.{ }^{64}\right)$ Semler, NZG 2007, $883 \mathrm{mwN}$.

${ }^{65}$ ) Beide haften der Gesellschaft nach Ansicht des BGH gesamtschuldnerisch; BGHZ 168, 195; zustimmend Vetter, ZIP 2008, 10

${ }^{66}$ ) Zu Besonderheiten des Anspruchs Lutter, FS Harm Peter Westermann 1186 (Verdrängung von $\S 818$ Abs 3 BGB und $\S 814$ BGB).

${ }^{67}$ ) So $\mathrm{zB}$ in der Entscheidung des LG Stuttgart 27.5.1998, BB 1998, 1549 ff.

\section{Regel 49 CGK}

In Österreich wurde mit Regel 49 CGK die Problematik 2002 erstmals geregelt:

„Verträge, insbesondere Beratungsverträge des Unternehmens mit einzelnen Mitgliedern des Aufsichtsrats bzw. ihnen nahe stehenden Unternehmen, bedürfen der Zustimmung des gesamten Aufsichtsrats, mit Ausnahme von Geschäften des täglichen Lebens. Gegenstand derartiger Verträge und Entgelt werden im Geschäftsbericht veröffentlicht.“68)

Mit Einführung des $\S 95$ Abs 5 Z 12 AktG durch das GesRÄG $2005 \mathrm{kam}$ es zur Überarbeitung des CGK. Regel 48 entspricht nun dem Gesetzeswortlaut ${ }^{69}$ ); die neue Regel 49 behält die Pflicht zur Veröffentlichung im Geschäftsbericht bei ${ }^{70}$ ). Interessant war die Interpretation der Regel $49 \mathrm{aF}$ durch den Arbeitskreis für Corporate Governance ${ }^{71}$ ):

„Die Bestimmung erfasst Beratungsverträge und andere Verträge des Aufsichtsratsmitgliedes nicht nur mit der Gesellschaft selbst, sondern auch mit Konzernunternehmen. Ebenso [...] Verträge [...] mit Unternehmen, an denen das Aufsichtsratsmitglied beteiligt ist (etwa Partner in einer Rechtsanwaltsgesellschaft) oder in denen das Aufsichtsratsmitglied eine Organfunktion ausübt (etwa Vorstandsdirektor der Hausbank). Hinsichtlich der Unternehmen, an denen das Aufsichtsratsmitglied beteiligt ist oder in denen es eine Organfunktion ausübt, ist Voraussetzung, dass die Organfunktion und die Beteiligung geeignet ist, wesentlichen Einfluss auf die Gesellschaft auszuüben. [...]“72)

Die Interpretation war weit, was die Einbeziehung von Verträgen mit Konzernunternehmen (zB Tochtergesellschaften) unter ,Verträge des Unternehmens" betrifft. Sonst war sie aber überra-

${ }^{68}$ ) Fassung vom 22.2.2005. Dabei handelte es sich um eine sog C-Regel, bei der also die Befolgung nicht zwingend war, Abweichungen aber zu begründen waren. $\mathrm{S}$ Haberer, Corporate Governance $131 \mathrm{ff}$.

$\left.{ }^{69}\right)$ Stand Juni 2007.

${ }^{70}$ ) Bei der Veröffentlichung ist die Zusammenfassung gleichartiger Verträge zulässig. Regel 48 ist eine „L-Regel“ (Befolgung zwingend), während Veröffentlichung von Gegenstand und Entgelt (Regel 49) auch weiterhin eine C-Regel ist (begründete Abweichung zulässig). Zur Veröffentlichungspflicht in Deutschland s 5.5.4. dCGK; dazu Kremer in Ringleb/Kremer/Lutter/v. Werder, Corporate Governance ${ }^{2} \mathrm{Rz} 1125$ ff; einschränkend Kropff in Semler/v. Schenck, Arbeitshandbuch ${ }^{2} \S 8 \quad \mathrm{Rz} 109$ (FN 158).

$\left.{ }^{71}\right)$ Ebenso Reich-Rohrwig, Erweiterung der AR-zustimmungspflichtigen Geschäfte, ecolex 2006, 35

${ }^{72}$ ) Interpretationen des CGK sind im Internet abrufbar (http://www.corporate-governance.at); zuletzt besucht am 8.2.2008. Auf der Homepage der Arbeitsgruppe für Corporate Governance findet sich der Hinweis: „Es handelt sich dabei um im Arbeitskreis abgestimmte Interpretationen, die als Umsetzungshilfe gedacht sind. Diese Interpretationen sind nicht abschließend, ihre Beachtung gewährleistet aber jedenfalls ein kodexkonformes Verhalten". Auch sonst deutete die Formulierung der Interpretation nicht auf die Möglichkeit eines weiteren Verständnisses hin - den von der oben zitierten Interpretation genannten Fällen geht keine Bezeichnung wie ,zB“ oder ,,insb“ voran. Offensichtlich wollte der Arbeitskreis demnach keine anderen als die in seiner Interpretation genannten Konstellationen erfasst sehen. 
schend eng: Von zentraler Bedeutung war die stark einschränkende Interpretation des ,nahestehenden Unternehmens“. Zunächst wurden darunter alle Unternehmen verstanden, an denen Aufsichtsratsmitglieder beteiligt sind oder in denen sie Organfunktionen ausüben ${ }^{73}$ ). Schon der erste Teil der Interpretation, der das Naheverhältnis auf Beteiligung oder Organmitgliedschaft beschränkt, erscheint verfehlt. Denn nach diesem Verständnis stünde ein Unternehmen dem Aufsichtsratsmitglied zB nicht „nahe“, wenn seine Ehegattin sämtliche Anteile daran hält oder es zu diesem in permanenter, umfassender Konsulentenbeziehung steht; nicht einmal dann stand das Aufsichtsratsmitglied einem Unternehmen „nahe“, wenn dieses sein Arbeitgeber war, aber das Aufsichtsratsmitglied nicht im Vorstand (Organmitglied), sondern als leitender Angestellter (etwa mit ergebnisabhängiger Prämie) tätig war. Überraschend ist schließlich, dass an die Privatstiftung im Zusammenhang mit der Interpretation von „nahestehend" offenbar nicht gedacht wurde.

Der Arbeitskreis reduzierte aber den Anwendungsbereich in einem zweiten Schritt noch weiter: Organstellung und Beteiligung führten nur zur Anwendung der Regel, wenn damit auch ein erheblicher Einfluss verbunden war. Dieses Verständnis verfehlt das Regelungsziel (Vermeidung unsachlicher Einflüsse) und geht an der Realität vorbei. Denn auch eine geringe Beteiligungshöhe, die gar keinen Einfluss verschafft, kann einen erheblichen absoluten Wert haben. Der Aufsichtsrat der Gesellschaft A hat wegen § 95 Abs 5 AktG oder eines Zustimmungsvorbehalts ${ }^{74}$ ) über die Vergabe eines Großauftrags an die Gesellschaft B, C oder D oder über die Auswahl der Bank B, C oder D für die Begleitung einer Kapitalerhöhung zu entscheiden. Ist ein Aufsichtsratsmitglied an der Gesellschaft B beteiligt, können erhebliche finanzielle Interessen die Entscheidungsfindung - bewusst oder unbewusst - beeinflussen. Einer Beteiligung des Aufsichtsratsmitglieds an B, die einen erheblichen Einfluss verschafft, bedarf es für die Entstehung von Eigeninteressen nicht.

\section{Anwendungsbereich von $\S 95$ Abs $5 \mathrm{Z} 12$ AktG (und § 30j Abs 5 Z 10 GmbHG)}

„Maßnahmen der Geschäftsführung können dem Aufsichtsrat nicht übertragen werden. Folgende Geschäfte sollen jedoch nur mit Zustimmung des Aufsichtsrats vorgenommen werden: [...]

12. der Abschluss von Verträgen mit Mitgliedern des Aufsichtsrats, durch die sich diese außerhalb ihrer Tätigkeit im Aufsichtsrat gegenüber der Gesellschaft oder einem Tochterunternehmen (§ 228 Abs. 3 UGB) zu einer

$\left.{ }^{73}\right)$ Nach Ch. Nowotny, Neues zum Aufsichtsrat, RdW 2005, 660 habe die Erfassung von Personen mit Organfunktionen nur Vorstände oder Geschäftsführer, nicht aber Aufsichtsratsmitglieder gemeint.

${ }^{74}$ ) Zum Beurteilungsspielraum bei Zustimmungsvorbehalten vor dem Hintergrund der ARAG-Entscheidung des BGH s $M$. Roth, GesRZ-Sonderheft Oktober 2005, 17; Semler, NZG 2007, 882.
Leistung gegen ein nicht bloß geringfügiges Entgelt verpflichten. Dies gilt auch für Verträge mit Unternehmen, an denen ein Aufsichtsratsmitglied ein erhebliches wirtschaftliches Interesse hat."

\section{Einschränkungen (insb auf börsenotierte AG) entfallen}

Die Regel gilt auch für nicht notierte AG und für jede $\mathrm{GmbH}$, bei der ein Aufsichtsrat eingerichtet wurde. Der Anwendungsbereich ist damit erheblich weiter als im CGK. Im Übrigen ist die Anwendbarkeit auch für Geschäfte mit einer Privatstiftung, auf die ein Aufsichtsratsmitglied erheblichen Einfluss als Stifter oder Organmitglied hat oder zu deren Begünstigten es zählt, anzunehmen $\left.^{75}\right)$.

\section{Vor Amtsantritt des Aufsichtsratsmitglieds ab- geschlossene Verträge}

Unklar ist, ob die Zustimmung nach $\S 95$ Abs 5 Z 12 AktG notwendig ist, wenn die Vereinbarung vor der Bestellung des Aufsichtsratsmitglieds abgeschlossen wurde. In Deutschland wird das überwiegend bejaht ${ }^{76}$ ). Die Situation ist in Österreich wegen $\S 87$ Abs 1a AktG teilweise anders: Personen, die zur Wahl in den Aufsichtsrat antreten ${ }^{77}$ ), müssen der Hauptversammlung vorher ihre fachliche Qualifikation, ihre beruflichen Funktionen sowie alle Umstände, die die Besorgnis einer Befangenheit begründen könnten, darlegen ${ }^{78}$ ). Soweit es um bereits erfüllte Verträge der Gesellschaft mit zukünftigen Aufsichtsratsmitgliedern geht, wird die Besorgnis der Befangenheit kaum entstehen. Primär geht es um nicht erfüllte Verträge mit der Gesellschaft sowie Verträge mit Unternehmen, an denen das Aufsichtsratsmitglied ein erhebliches wirtschaftliches Interesse hat. Wird die Hauptversammlung informiert und erfolgt danach die Wahl, ist die Kontrolle durch den Aufsichtsrat überflüs$\operatorname{sig}^{79}$ ): Risiken der Vorteilszuwendung bestehen nicht, soweit das Geschäft vor der Bestellung in den Aufsichtsrat geschlossen wurde (Ausnahme:

${ }^{75}$ ) S auch Fida, Zur Genehmigungspflicht von Sonderverträgen mit Aufsichtsratsmitgliedern, wbl 2006, 360 .

$\left.{ }^{76}\right) \mathrm{Hopt} / \mathrm{M}$. Roth, $\mathrm{AktG}^{4} \S 114 \mathrm{Rz} 31$; Semler in MünchKomm AktG ¿ $_{114} \mathrm{Rz} 54 ;$ Hüffer, AktG ${ }^{7} \S 114 \mathrm{Rz} 2$; Kropff in Semler/v. Schenck, Arbeitshandbuch ${ }^{2}$ \& 8 Rz 121; Vetter, ZIP 2008, 9 f.

${ }^{77}$ ) Vgl Fida/Hofmann, Neue Verfahrensvorschriften für die Wahl in den Aufsichtsrat, Aufsichtsrat aktuell 2006, $12 \mathrm{f}$.

${ }^{78}$ ) Im Hinblick auf die Zielsetzung der Bestimmung wäre es sinnvoll gewesen, die Informationspflichten auch bei späterer Befangenheit ausdrücklich vorzuschreiben. Beispiel: Ein Aufsichtsratsmitglied gelangt durch einen Unternehmenserwerb während seiner Funktionsperiode in eine vergleichbare Situation. Eine Informationspflicht bei der nächsten ordentlichen Hauptversammlung wäre sinnvoll. Haben $10 \%$ der Aktionäre Bedenken, könnten sie die Abberufung des Aufsichtsratsmitglieds betreiben (gerichtliche Abberufung bei wichtigem Grund auf Antrag von $10 \%$ des Grundkapitals gem $\S 87 \mathrm{Abs} 5 \mathrm{AktG}$; oder 3/4-Mehrheit gem $\S 87$ Abs 3 AktG).

${ }^{79}$ ) Ebenso Fida, wbl 2006, 359. 
Dauerschuldverhältnisse) ${ }^{80}$ ). Die Information der Hauptversammlung schafft auch Transparenz gegenüber den anderen Aufsichtsratsmitgliedern ${ }^{81}$ ).

Eine Zustimmung des Aufsichtsrats ist allerdings bei vor Amtsantritt geschlossenen Vereinbarungen notwendig, wenn die Hauptversammlung entgegen $\S 87$ Abs 1a AktG nicht informiert wurde. Denn die Information der Hauptversammlung vor der Wahl dient den gleichen Zielen wie die Kontrolle und Transparenz durch $\S 95$ Abs 5 Z 12 AktG. Bei nicht erfüllten Verträgen zwischen Gesellschaft und neu gewähltem Aufsichtsratsmitglied führt der Verstoß gegen $\S 87$ Abs 1a AktG daher $\mathrm{mE}$ nachträglich zur Anwendung von $\S 95$ Abs $5 \mathrm{Z} 12$ AktG. In der Praxis empfiehlt sich die korrekte Information der Hauptversammlung vor der Wahl.

\section{Unterschiede zu Regel 49 CGK aF}

Anders als in $\S 114$ dAktG bedurften Verträge mit Unternehmen, die einem Aufsichtsratsmitglied nahestanden, in Österreich bereits nach dem Wortlaut von Regel 49 CGK aF eines Aufsichtsratsbeschlusses. Nach $\S 95$ Abs 5 Z 12 AktG gilt das nun für Verträge mit Unternehmen, an denen das Aufsichtsratsmitglied ein erhebliches wirtschaftliches Interesse hat. Dabei besteht allerdings ein wichtiger Unterschied zu Regel 49 CGK aF. Die oben kritisch hinterfragte Interpretation des Arbeitskreises für Corporate Governance ist wegen der Gesetzesformulierung nicht mehr relevant ${ }^{82}$ ). Ausschlaggebend ist nun das erhebliche wirtschaftliche Interesse. Dieses ist $\mathrm{zB}$ vorhanden, wenn ein Aufsichtsratsmitglied an einem Unternehmen (mit dem die AG einen größeren Vertrag abschließt) beteiligt ist und die Beteiligung mehr als einen bloß geringfügigen Wert hat. Eines erheblichen Einflusses bedarf es nicht - auch bei geringer Beteiligungshöhe kann ein erhebliches wirtschaftliches Interesse entstehen ${ }^{83}$ ). Nur wenn die Werte in absoluter Höhe und im Verhältnis $\mathrm{zu}$ den Begleitumständen geringfügig sind, fehlt das erhebliche wirtschaftliche Interesse. Diese Sichtweise wird durch den neuen Grundtatbestand un-

$\left.{ }^{80}\right)$ UU anders sind vor Amtsantritt abgeschlossene Dauerschuldverhältnisse zu beurteilen. Während beim abgeschlossenen Zielschuldverhältnis das Aufsichtsratsmitglied bei seiner Bestellung einen definierten Anspruch hat, kann der Vorstand beim Dauerschuldverhältnis kündigen und die vor Bestellung begründete Vorteilszuwendung beenden. Durch diesen Zustand wird eine zusätzliche Abhängigkeit geschaffen, die die Befassung des Aufsichtsrats gem $\S 95$ Abs 5 Z 12 AktG ( $\$ 30$ Abs 5 Z $10 \mathrm{GmbHG)} \mathrm{rechtfertigt:} \mathrm{Wenn} \mathrm{die} \mathrm{Gesellschaft} \mathrm{das}$ Vertragsverhältnis auch nach Veränderung der Umstände (Marktumfeld), die zur Unangemessenheit des Leistungsverhältnisses führen, nicht beendet, kann sich daraus ein unangemessener Vorteil ergeben.

${ }^{81}$ ) Sie sollten in der Hauptversammlung anwesend sein (§ 102 Abs 2 AktG).

${ }^{82}$ ) Ebenso Kalss, Aufsichtsrat aktuell 2006, 7.

$\left.{ }^{83}\right)$ Einschränkend jedoch Kalss, SWK 2006, 422; Fida, wbl 2006, 359 f. S hingegen Peltzer, ZIP 2007, 306. termauert, der auf ein „nicht bloß geringfügiges Entgelt" abstellt (womit auch die durchgerechnete Quote bei einer Beteiligung gemeint ist). Die Gesetzesmaterialien stellen sogar auf den bloßen Anschein der Befangenheit ab.

In dieselbe Richtung geht eine jüngere Entscheidung des $\mathrm{BGH}^{84}$ ). In der mündlichen Verhandlung hatte der Senatsvorsitzende Prof. Goette folgendes Beispiel gebildet ${ }^{85}$ ): Ein Aufsichtsratsmitglied ist mit $1 \%$ an einer Beratungsgesellschaft beteiligt, die von der AG ein Beratungshonorar von $€ 1$ Mio erhält. Die auf das Aufsichtsratsmitglied entfallenden $€ 10.000$,- können nach Auffassung Goettes zur Anwendung von $\S 114 \mathrm{dAktG}$ führen. Die in Folge dieser Gerichtsverhandlung ergangene Entscheidung hat in Deutschland für Aufsehen gesorgt. Das neuere deutsche Schrifttum stellt nun meist auf die persönlichen finanziellen Anreize des betroffenen Aufsichtsratsmitglieds $a b^{86}$ ).

\section{Finanzielles Interesse auch durch Geschäfts- führertätigkeit}

Neben direkten und persönlichen finanziellen Vorteilen kann das erhebliche wirtschaftliche Interesse auch durch Organfunktion in dem kontrahierenden Unternehmen entstehen (selbst wenn die finanziellen Vorteile nicht in der Person des betroffenen Organmitglieds, sondern in der Gesellschaft entstehen ${ }^{87}$ ). Beispiel: Wird ein Versicherungsvertrag der AG mit einer Versicherung geschlossen, deren Vorstand im Aufsichtsrat der AG sitzt, liegt ein erhebliches wirtschaftliches Interesse vor, und der Vertrag ist genehmigungspflichtig. Jedenfalls gilt das für Geschäftsführer und Vorstände. Der Wortlaut erfasst auch Aufsichtsratsmitglieder ${ }^{88}$ ).

\section{V. „Nicht bloß geringfügiges Entgelt“"}

Nur Leistungsverpflichtungen, die gegen ein nicht bloß geringfügiges Entgelt erbracht werden, sind von der neuen Bestimmung erfasst. Gegenüber der Regel 49 CGK aF (Ausnahme nur für "Geschäfte des täglichen Lebens") besteht insoweit eine Lockerung. Zunächst scheidet die Anwendung auf unentgeltliche Leistungen des Aufsichtsratsmitglieds aus ${ }^{89}$ ). Offen ist, ab wann ein

\footnotetext{
${ }^{84}$ ) BGH ZIP 2007, 22.

$\left.{ }^{85}\right)$ Nachweise bei Peltzer, ZIP 2007, 306 FN 7

$\left.{ }^{86}\right)$ Dazu Peltzer, ZIP 2007, 306: Es ,[...] ist die Höhe der Beteiligung [...] nicht entscheidend, sondern es kommt vielmehr auf die Höhe der Vergütung an, die auf das Aufsichtsratsmitglied entfällt. Nur wenn diese ganz und gar marginal und zwar absolut oder im Verhältnis $\mathrm{zu}$ der statutarischen Aufsichtsratsvergütung ist, kommen $\S \S 113$ und 114 AktG nicht zur Anwendung". S auch Vetter, AG 2006, 177; Werner, DB 2006, 936; Benecke, WM 2007, 719; Vetter, ZIP 2008, 8 f.

$\left.{ }^{87}\right)$ Ebenso Ch. Nowotny, RdW 2005, 660 und Lutter, FS Harm Peter Westermann $1180 \mathrm{f}$.

${ }^{88}$ ) $\mathrm{S}$ aber die Ansicht zum CGK aF von Ch. Nowotny, RdW 2005, 660 .

${ }^{89}$ ) Egermann, GesRÄG 2005 - Zu den geplanten Änderungen beim Aufsichtsrat, RdW 2005, 70.
} 
Entgelt nicht mehr bloß geringfügig ist. Das wird $\mathrm{mE}$ vor dem Hintergrund der Aufsichtsratsvergütung zu beurteilen sein ${ }^{90}$ ). Im internationalen Vergleich sind österr Aufsichtsratsvergütungen oft sehr niedrig - vielfach liegen sie in der Größenordnung von $€ 5.000,-$ bis $€ 10.000,-$ pro Jahr ${ }^{91}$ ). Daher erscheint, je nach den Verhältnissen der Gesellschaft, eine Geringfügigkeitsgrenze von etwa $€ 500,-$ bis $€ 1.000$,- sachgerecht ${ }^{92}$ ); dies entspricht auch den üblichen Obergrenzen für Sitzungsgelder pro Sitzung.

Bei Unklarheit darüber, ob ein Entgelt bloß geringfügig ist oder nicht, wird es sinnvoll sein, die Zustimmung des Aufsichtsrats einzuholen ${ }^{93}$ ). Eine klarstellende Festlegung eines Betrages, ab dem die Geringfügigkeit überschritten ist, kann in der Satzung oder in der Geschäftsordnung des Aufsichtsrats erfolgen $^{94}$ ). Jedoch ist $\S 95$ Abs 2 Z 12 AktG zwingend - Satzung und Geschäftsordnung des Aufsichtsrats haben daher nur wenig Spielraum. Die gesetzliche Wertung können sie nicht verdrängen (ein Freiraum von $\mathrm{zB} € 10.000$,- kann also sicher nicht wirksam festgelegt werden) ${ }^{95}$ ).

\section{Gleichstellung von Verträgen des Aufsichts- ratsmitglieds mit Konzernunternehmen (Toch- terunternehmen, Mutterunternehmen, Schwes- terunternehmen etc)}

$\S 95$ Abs 5 Z 12 AktG erfasst schon dem Wortlaut nach nicht nur Verträge eines Aufsichtsratsmitglieds mit der beaufsichtigten Gesellschaft selbst, sondern auch mit deren Tochterunternehmen. Das verhindert Umgehungen ${ }^{96}$ ). In seiner Interpre-

\footnotetext{
$\left.{ }^{90}\right)$ S auch BGH 20.11.2006, ZIP 2007, 22.

$\left.{ }^{91}\right)$ Nachweise bei Egermann, RdW 2005, $70 \mathrm{FN} 47$

$\left.{ }^{92}\right)$ Etwas großzügiger ist der Vorschlag einer Grenze von $€ 5.000$,- von Ch. Nowotny, RdW 2005, 660, der dafür auf die entsprechende Grenze des $\S 28$ BWG für Organgeschäfte verweist; ihm folgend offenbar Fida, wbl 2006, 358. Das erscheint $\mathrm{mE} z u$ hoch. Denn auch bei einer strengeren - also niedrigeren - Grenze wäre wohl nicht zu befürchten, dass dem Aufsichtsrat zahllose zusätzliche Geschäfte mit Aufsichtsratsmitgliedern zur Genehmigung vorgelegt werden müssten (welche Geschäfte sollten dies sein?). Unerträglicher zusätzlicher Aufwand ist also nicht zu befürchten. Nach Egermann, RdW 2005, 70 könne sogar ab $€ 500,-$,keinesfalls mehr von einem geringfügigen Entgelt gesprochen werden"; sehr streng auch Semler, NZG 2007, 886, der (in Österreich) bis zur Klärung des Begriffs „Geringfügigkeit" als Berater stets die Zustimmung einholen würde. Vgl für Deutschland Vetter, ZIP 2008, 9.

${ }^{93}$ ) Kalss in Doralt/Nowotny/Kalss, AktG \& $95 \mathrm{Rz} 98$.

$\left.{ }^{94}\right)$ So bereits Kastner, Insolvenzrecht und Gesellschaftsrecht, GesRZ 1982, 216 (§ 95 AktG idF des GesRÄG 1982, bezüglich der neu eingeführten zustimmungspflichtigen Geschäfte). S auch Kalss, SWK 2006, 420, 425; Fida, wbl 2006, 358.

95) Ähnlich Fida, wbl 2006, 358.

${ }^{96}$ ) Für den Begriff der Tochtergesellschaften wird auf $\S 228$ Abs 3 HGB verwiesen; dazu Ch. Nowotny in Straube (Hrsg), HGB Kommentar, Band II Rechnungslegung ${ }^{2}$ (2000) § $228 \mathrm{Rz} 40$ ff und $\S 244 \mathrm{Rz} 2 \mathrm{f}$.
}

tation $^{97}$ ) von Regel 49 CGK aF nahm der Arbeitskreis für Corporate Governance an, dass unter dem Begriff ,Unternehmen“ alle Konzerngesellschaften, auch Muttergesellschaften zu verstehen sind. Nach dem bloßen Wortlaut der neuen Regelung spricht nun ein Umkehrschluss gegen diese Interpretation. Dem Zweck der Vorschrift entspricht der Umkehrschluss wohl nur partiell. Ist der Vertrag mit der Muttergesellschaft dem Aufsichtsrat der Tochter bekannt und ist strukturell daraus keine Abhängigkeit des Aufsichtsratsmitglieds vom Tochtervorstand zu befürchten, bedarf es wohl keiner Genehmigung des Aufsichtsrats ${ }^{98}$ ). Beispiele: Dienstvertrag des Vorstandsmitglieds der Muttergesellschaft, das auch im Aufsichtsrat der Tochtergesellschaft tätig ist; Mandatsverhältnis eines Aufsichtsratsmitglieds der Muttergesellschaft. Andernfalls würde auch die Zuständigkeitsordnung für Dienstverträge und für Aufsichtsratsvergütungen im Konzern gestört. Ein zusätzlicher Vertrag zwischen Muttergesellschaft und konzernzugehörigem Aufsichtsratsmitglied sollte aber dem Aufsichtsrat nach der allgemeinen Regel zur Genehmigung vorgelegt werden. Denn es ist gut denkbar, dass gerade zur Umgehung der Kompetenz des Aufsichtsrats der Gesellschaft und zur Vermeidung der nach dem Gesetz gewünschten Transparenz der Vorstand der Muttergesellschaft, möglicherweise im Einvernehmen mit dem Vorstand der Tochtergesellschaft, einen Vertrag mit einem Aufsichtsratsmitglied der (Tochter-)Gesellschaft schließt; damit leidet die Kontrollfunktion genauso wie bei einem Vertrag zwischen Aufsichtsratsmitglied und Gesellschaft $\left.{ }^{99}\right)$. Die Beurteilung, ob der Vertrag im Interesse der Gesellschaft liegt, sollte in diesen Fällen dem Aufsichtsrat obliegen. Das gilt natürlich erst recht, wenn zwischen Vorstand der Mutter- und

$\left.{ }^{97}\right)$ S C.II.

$\left.{ }^{98}\right)$ Lutter, FS Harm Peter Westermann 1185; Vetter, ZIP 2008, 9

${ }^{99}$ ) AA offenbar Lutter, FS Harm Peter Westermann 1185, der in diesem Zusammenhang keine der spezifischen Gefahren, die mit $\S 114$ dAktG verhindert werden sollen, sieht, weil der Vorstand der Tochter auf den Vertragsschluss zwischen eigenem Aufsichtsratsmitglied und der Mutter keinen Einfluss nehmen könne. Bei einem Kernaktionär mit Beteiligung an der Konzernspitze beschränkt sich der Einfluss allerdings selten auf die Konzernobergesellschaft (insb bei größeren Familienunternehmen); auch in den Tochtergesellschaften werden Vorstands- und Aufsichtsratsmandate regelmäßig mit Personen besetzt, zu denen ein besonderes Vertrauens- und Naheverhältnis besteht. Das schafft $\mathrm{mE}$ wechselseitige Abhängigkeiten und Informationskanäle, die die Möglichkeiten der Einflussnahme eröffnen und auch eine Basis für Umgehungen durch Verträge des Aufsichtsratsmitglieds mit der Mutter (statt direkt mit der Tochter) schaffen. Darüber hinaus besteht eine der spezifischen Gefahren, denen das Gesetz zu begegnen sucht, wenn der Vorstand der Mutter auch im Aufsichtsrat der Tochter ist und (eventuell gegen die Interessen der Minderheitsgesellschafter der Tochter) ein Aufsichtsratsmitglied beeinflussen will. 
Vorstand der Tochtergesellschaft personelle Identität besteht ${ }^{100}$ ).

\section{VII. ,[... ] gegenüber der Gesellschaft [...] zu einer Leistung [...] verpflichten“"}

Durch die weite Formulierung werden - anders als in Deutschland - nicht nur Beratungsverträge, sondern auch sonstige Verträge erfasst: $§ 95$ Abs 5 Z 12 AktG gilt für alle Verträge mit einem Unternehmen, an dem ,das Aufsichtsratsmitglied ein erhebliches wirtschaftliches Interesse hat". Geht ein Unternehmen, an dem das Aufsichtsratsmitglied ein erhebliches wirtschaftliches Interesse hat, eine Geschäftsbeziehung zur Gesellschaft ein, ist $\S 95$ Abs 5 Z 12 AktG anwendbar (Vertrag mit einem Lieferanten, einer Bank, einem Versicherungsunternehmen etc).

Bei diesen in Österreich erfassten Verträgen drohen zwar meist nicht die geschilderten spezifischen Probleme des durch die Beratung voreingenommenen Aufsichtsratsmitglieds, aber es droht das Problem finanzieller Anreize und Abhängigkeiten, da selbst Geschäfte zu marktüblichen Konditionen Gewinnchancen vermitteln ${ }^{101}$ ). Hinzu kommt, dass Sondervorteile an Aufsichtsratsmitglieder in Form verdeckter Zuwendungen nur schwer nachvollziehbar sind ${ }^{102}$ ). Selbst wenn über das Geschäft informiert wird, kann ein branchenfremdes Aufsichtsratsmitglied die Überhöhung des Entgelts kaum erkennen. Praktisch ist Aufsichtsratsmitgliedern zu raten, bei Information über derartige Geschäfte die Zusicherung der Marktüblichkeit der Bedingungen zu verlangen ${ }^{103}$ ). Auch bei angemessenem Entgelt kann der Aufsichtsrat aber die Genehmigung verweigern (insb wenn Abhängigkeiten drohen). Die Entscheidung hat sich daran $\mathrm{zu}$ orientieren, ob der $\mathrm{zu}$ genehmigende Vertrag im Interesse der Gesellschaft liegt.

Nach der Gesetzesformulierung erfasst $\S 95$ Abs $5 \mathrm{Z} 12 \mathrm{~S} 1$ AktG nur Verträge, in denen sich das Aufsichtsratsmitglied gegenüber der Gesellschaft „zu einer Leistung gegen [...] Entgelt" verpflichtet $\left.^{104}\right)$. Auch wenn die Gegenüberstellung der Begriffe „Leistung“ und „Entgelt" zunächst suggeriert, dass der Leistung des Aufsichtsratsmitglieds (zB Lieferung oder Werkleistung) eine Geldzahlung der AG gegenübersteht, gestattet es der Sprachgebrauch, auch Geldzahlungen als Leistungen (Geldleistungen) zu qualifizieren; ebenso können zB Sachleistungen als Entgelt qualifiziert wer-

\footnotetext{
${ }^{100}$ ) Vetter, ZIP 2008, 9.

101) Vetter, ZIP 2008, 6

$\left.{ }^{102}\right)$ Darauf verweist auch Reich-Rohrwig, ecolex 2006, 36.

${ }^{103}$ ) Wird diese Zusicherung gegeben, ist sicher nur bei konkreten Anhaltspunkten für Zweifel eine Nachprüfung notwendig. Bei überhöhtem Entgelt wäre die Genehmigung in aller Regel zu verweigern, weil eine nicht fremdübliche Gegenleistung mit dem Wohl des Unternehmens nicht $\mathrm{zu}$ vereinbaren ist (Verstoß gegen $\S 70$ AktG iVm § 84 AktG und § 100 AktG).

${ }^{104}$ ) Vgl Ch. Nowotny, RdW 2005, 659 .
}

den. Es lässt sich daher schon nach dem Wortlaut der Bestimmung argumentieren, dass es nicht darauf ankommt, ob das Aufsichtsratsmitglied etwas leistet bzw liefert und die AG dafür bezahlt, weil auch eine "Geldleistung“ des Aufsichtsratsmitglieds gegen ein zB in Waren bestehendes Entgelt der AG unter den Wortlaut subsumierbar ist. Erst recht folgt das aus dem Zweck der Norm, die im Vergleich $\mathrm{zu}$ ihrem deutschen Vorbild viel weiter gefasst ist. Für das Ziel der Norm, Vertragsbeziehungen der Aufsichtsratsmitglieder mit der Gesellschaft an die Zustimmung des Aufsichtsrats $\mathrm{zu}$ binden und verdeckte Zuwendungen zu vermeiden, ist es gleichgültig, wer leistet und wer Geld bezahlt. Wäre $\S 95$ Abs $5 \mathrm{Z} 12$ AktG bloß auf die entgeltliche Beratungsleistung des Aufsichtsratsmitglieds zugeschnitten, könnte die Beschränkung auf (Beratungs-)Leistungen des Aufsichtsratsmitglieds überzeugen. Da aber in Österreich jede Art von „Leistung“ erfasst wird, wäre es schwer begründbar, ausschließlich auf Leistungen des Aufsichtsratsmitglieds gegenüber der Gesellschaft abzustellen. Die anfangs geschilderten Risiken sind ebenso denkbar, wenn nicht das Aufsichtsratsmitglied, sondern die Gesellschaft die Leistung erbringt. Ebenso wird die Unabhängigkeit gefährdet, wenn die Gesellschaft an ein Unternehmen leistet, an dem das Aufsichtsratsmitglied ein erhebliches wirtschaftliches Interesse hat. Wird die Leistung nicht zu einem marktüblichen Entgelt erbracht, liegt die Vorteilszuwendung auf der Hand. Meist wird damit auch ein Nachteil der Gesellschaft verbunden sein $\left.^{105}\right)$. Im Ergebnis ist $\S 95$ Abs 5 Z 12 AktG auf Leistungen der Gesellschaft gegenüber dem Aufsichtsratsmitglied analog anzuwenden ${ }^{106}$ ).

\section{Verträge im Konzern}

Nach dem Wortlaut unterliegen $\S 95$ Abs 5 Z 12 AktG auch Geschäfte im faktischen Konzern, wenn $\mathrm{zB}$ ein Aufsichtsratsmitglied und Kernaktionär Vertragspartner der AG ist. Das ergibt sich aus der klaren Gleichstellung von Verträgen mit dem Aufsichtsratsmitglied und Verträgen mit Unternehmen, an denen das Aufsichtsratsmitglied ein erhebliches wirtschaftliches Interesse hat. Ob das sinnvoll ist, hängt einerseits von der konkreten Situation ab: So wird bei einer hundertprozentigen Tochtergesellschaft und deren Geschäften mit der Muttergesellschaft im Normalfall kein Bedarf für eine derartige Kontrolle der Konzerngeschäfte bestehen. Die typischen Gefahrenmomente, für die $\S 95$ Abs $5 \mathrm{Z} 12$ AktG eingeführt wurde, sind nicht gegeben. Bedenken gegen die Anwendung auf Konzerngeschäfte bestehen auch wegen der feh-

${ }^{105}$ ) Der Nachteil der Gesellschaft ist allerdings nicht zwingend. Auch eine zu billig erbrachte Leistung, die dem Aufsichtsratsmitglied einen Sondervorteil bringt, kann für die Gesellschaft noch immer ein gewinnbringendes Geschäft sein.

${ }^{106}$ ) Ebenso Ch. Nowotny, RdW 2005, 660; Fida, wbl 2006,357 . 
lenden Praktikabilität der Genehmigung jedes Konzerngeschäfts ${ }^{107}$ ).

Andererseits hängt die Frage der Anwendung auf Konzerngeschäfte davon ab, welchen Zweck $\S 95$ Abs 5 Z 12 AktG erfüllen soll und ob der Aufsichtsrat auch für typische Konzernprobleme stärker in die Pflicht genommen werden soll. Die Anwendung auf Konzerngeschäfte ${ }^{108}$ ) kann bei Tochtergesellschaften mit Minderheitsgesellschaftern eine Kontrolle bieten und konzernfreie Aktionäre vor Verletzung des at-arms-length-Prinzips bei Konzerngeschäften bewahren. Damit wäre der Gleichbehandlung aller Aktionäre gedient. Soweit es allerdings um Geschäfte mit hundertprozentigen Tochtergesellschaften im Konzern geht, erscheint die Gesetzesbestimmung unglücklich weit geraten und ihre wortlautgetreue Anwendung nicht sinnvoll. $\mathrm{Zu}$ erwägen ist für diese Fälle eine teleologische Reduktion - für Konzerngeschäfte bestehen auch andere Schutzmechanismen ${ }^{109}$ ). Selbst ohne Reduktion könnte eine vermittelnde Lösung durch geringere Anforderungen an die Informationsdichte und -tiefe sowie großzügigere Handhabung von Vorratsbeschlüssen mit nachträglichen Berichtspflichten erreicht werden ${ }^{110}$ ).

\section{E. Rechtsfolgen}

Es überrascht, dass der österr Gesetzgeber sich zu den Rechtsfolgen nicht ausdrücklich geäußert hat. Möglicherweise war dem Gesetzgeber diese Schwäche bewusst. Für diese Annahme einer bewussten Regelung ohne ausdrückliche Rechtsfolgen spricht zwar, dass die Materialien auf das deutsche Vorbild verweisen, das ausdrücklich Rechtsfolgen anordnet. Allerdings findet sich in den Erläuternden Bemerkungen kein weiterführender Hinweis dazu, dass das deutsche Vorbild im Einzelnen näher analysiert wurde. Die Bestimmung bzw ihr Abweichen vom deutschen Vorbild ist wohl kein bewusstes Ergebnis aufwändiger Diskussionen. Zumindest findet sich dazu kein Anhaltspunkt in den äußerst knappen Erläuterungen ${ }^{111}$ ). Aus verschiedenen Gründen überrascht das auch

$\left.{ }^{107}\right)$ Kalss, SWK 2006, 423 ff; dieselbe, Aufsichtsrat aktuell 2006, 8 .

$\left.{ }^{108}\right)$ Für die Anwendung bei Konzerngeschäften ReichRohrwig, ecolex 2006, 36 .

109) Kastner/Doralt/Nowotny, Gesellschaftsrecht ${ }^{5}$ (1990) 241, 293 (FN 24); Peter Doralt in MünchKomm $\mathrm{AktG}^{2} \S 15 \mathrm{Rz} 51$; Peter Doralt in Doralt/Nowotny/Kalss, AktG $\S 15 \mathrm{Rz} 10$; Peter Doralt/Diregger in MünchKomm $\mathrm{AktG}^{2}$ IX/1 Österreichisches Konzernrecht Rz 48; Artmann, Gesellschaftsrechtliche Fragen der Organschaft zugleich ein Beitrag zum Konzernrecht (2004); Jabornegg in Jabornegg/Strasser (Hrsg), Kommentar zum Aktienge$\operatorname{setz}^{4}$ (2006) § $15 \mathrm{Rz} 37 \mathrm{ff}$ und Rz 43 ff; Artmann in Jabornegg/Strasser, $\mathrm{AktG}^{4} \S 52 \mathrm{Rz} 10 \mathrm{ff}$ und $\mathrm{Rz} 26$ ff; ReichRohrwig, Grundsatzfragen der Kapitalerhaltung bei $\mathrm{AG}, \mathrm{GmbH}$ sowie $\mathrm{GmbH}$ und Co KG (2004) $51 \mathrm{ff}, 93 \mathrm{ff}$; $U$. Torggler, Treuepflichten im faktischen Konzern (2007) 239 ff; Kalss/Eckert, Rechtsfragen von Geschäften im Konzern, RdW 2007, 583 ff.

${ }^{110)}$ Dazu bei E.III.

111) Erl RV 927 BlgNR 22. GP 8. nicht $\left.{ }^{112}\right)$. Dennoch lassen sich aus dem geltenden Recht verschiedene Rechtsfolgen ableiten.

\section{I. „Bringschuld“ sowohl des Vorstands als auch des Aufsichtsratsmitglieds}

Die Pflicht (und das Recht) zur Befassung des Aufsichtsrats mit einem Geschäft, das § 95 Abs 5 AktG unterliegt, trifft den Vorstand und das betroffene Aufsichtsratsmitglied ${ }^{113}$ ). Keiner der beiden kann sich damit entschuldigen, dass der andere die Information des Aufsichtsrats und die Herbeiführung des Beschlusses verabsäumt hat $\left.{ }^{114}\right)$.

\section{Erfordernis konkreter Information}

Soll ein Aufsichtsratsbeschluss die erwünschte Kontrolle bringen, so ist auch nach österr Recht zuvor die umfassende Information der anderen Aufsichtsratsmitglieder erforderlich ${ }^{115}$ ). Offenzulegen sind die konkrete Leistungsverpflichtung ${ }^{116}$ ), die Art der Leistung und ihr Umfang ${ }^{117}$ ). Diese Informationen sind schon deswegen notwendig, weil der Aufsichtsrat andernfalls nicht feststellen kann, ob die gegenständliche Leistung bereits im Rahmen der Aufsichtsratsfunktion geschuldet wäre ${ }^{118}$ ).

\footnotetext{
$\left.{ }^{112}\right)$ Einerseits war eine bereits bekannte Vorläuferbestimmung im CGK enthalten, deren Inhalt im Wesentlichen bloß ins Gesetz übernommen werden sollte. Andererseits hatten andere Bestimmungen, die mit demselben Gesetz beschlossen werden sollten, zu äußerst heftigen Auseinandersetzungen geführt. Weniger umstrittene Bestimmungen, wie die hier untersuchte, waren daher wohl nicht im Zentrum der Aufmerksamkeit.
}

${ }^{113}$ ) Enger (nur für eine Pflicht des Vorstands) Fida, wbl 2006, 358 f. Zur Vorlagepflicht des Vorstands bei zustimmungspflichtigen Geschäften nach § 95 Abs 5 AktG s Strasser in Jabornegg/Strasser, $\mathrm{AktG}^{4} \$ \S 95-97 \mathrm{Rz} 42$; Kalss in Doralt/Nowotny/Kalss, AktG $\S 95 \mathrm{Rz} 89$ (beide Kommentierungen beziehen sich allerdings auf die Rechtslage vor der Gesetzesergänzung des GesRÄG 2005, das in $\S 95$ Abs 5 AktG die neue $Z 12$ eingefügt hat). Vgl auch Kastner/Doralt/Nowotny, Gesellschaftsrecht $^{5}$, 261; Koppensteiner/Rüffler, GmbH-Gesetz Kom$\operatorname{mentar}^{3}(2007) \S 30 \mathrm{j} \mathrm{Rz} 18$.

$\left.{ }^{114}\right)$ Zur gleichgelagerten Frage nach deutschem Recht Semler in MünchKomm $\mathrm{AktG}^{2} \S 114 \mathrm{Rz} 54 ;$ Hüffer, $\mathrm{AktG}^{7}$ $\S 114 \mathrm{Rz} 6$; jeweils mwN

${ }^{115}$ ) Dazu bereits Hügel, GesRZ 1996, 216 ff lange vor den hier behandelten Änderungen des GesRÄG 2005. $\mathrm{Zu}$ den Informationsanforderungen bei § $95 \mathrm{Abs} 2 \mathrm{AktG}$ idF vor dem GesRÄG 2005 (also betreffend zustimmungspflichtiger Geschäfte im Allgemeinen): Krejci, Zur Berichtspflicht des AG-Vorstandes gegenüber dem Aufsichtsrat, FS Frotz (1993) 379 (mit hohen Anforderungen an die Informationspflichten des Vorstands gegenüber dem Aufsichtsrat).

${ }^{116)}$ Hopt/M. Roth, $\mathrm{AktG}^{4} \& 114 \mathrm{Rz} 52$ f; Kropff in Semler/v. Schenck, Arbeitshandbuch ${ }^{2} \S 8 \mathrm{Rz} 116 ;$ Mertens, $\mathrm{KK} \mathrm{AktG}^{2}$ \& $114 \mathrm{Rz} 6$

$\left.{ }^{117}\right)$ BGHZ 126, $344 \mathrm{f}$.

${ }^{118}$ ) S oben bei B. Wenn ja, muss der Aufsichtsrat seine Zustimmung verweigern; selbst wenn er sie erteilte, hätte das für die Wirksamkeit des Geschäfts keine Relevanz, weil nur die Hauptversammlung eine Sondervergütung für ein Aufsichtsratsmitglied beschließen kann (§ 98 AktG). Dazu Kalss in Doralt/Nowotny/Kalss, AktG $§ 98$ Rz 28; BGHZ 114, 127 ff; Semler in MünchKomm AktG $^{2}$ 
Dem Aufsichtsrat vorzulegen ist auch das Entgelt. Steht es im Voraus fest, ist die genaue Höhe zu nennen. Steht das Entgelt nicht fest, ist seine Berechnung so genau wie möglich offenzulegen; entsprechend der deutschen Vorbildbestimmung wird auch eine Obergrenze festzulegen $\operatorname{sein}^{119}$ ). Nur so kann gewährleistet werden, dass sich der Aufsichtsrat ein fundiertes Urteil über die Angemessenheit der Vergütung bildet ${ }^{120}$ ) (problematisch etwa im Fall Meinl Bank $\left.{ }^{121}\right)$ ). Auch für Österreich wird die Mitteilung, dass nach ,üblichen Stundensätzen“, berufsständischen oder gesetzlich geregelten Tarifen verrechnet würde, nicht genügen ${ }^{122}$ ).

\section{Beschlusserfordernis und Vorratsbeschlüsse}

Die Gesetzesformulierung lautet: ,[. . . ] sollen jedoch nur mit Zustimmung des Aufsichtsrats vorgenommen werden“. Daraus kann geschlossen werden, dass es nicht bloß um eine Informationspflicht geht - dann hätte der Gesetzeswortlaut etwa lauten müssen: „Der Aufsichtsrat ist über diese Verträge zu informieren" oder dgl. Wenn ein Geschäft nur mit Zustimmung des Aufsichtsrats erfolgen soll, bedarf es einer Willensbildung des Aufsichtsrats, also eines Beschlusses ${ }^{123}$ ). Die bloße Information oder eine unverbindliche Erörterung genügt nicht den Anforderungen von $\S 95$ Abs 5 AktG.

$\mathrm{Ob}$ auch sog Vorratsbeschlüsse ${ }^{124}$ ) genügen, ist fraglich ${ }^{125}$ ). Problematisch ist ein Vorratsbeschluss insb, wenn er lange vor Durchführung des Ge-

§ $114 \mathrm{Rz} 20$; Lutter/Kremer, ZGR 1992, 92; Kropff in Semler/v. Schenck, Arbeitshandbuch ${ }^{2} \S 8 \mathrm{Rz} 112$. Die in der Folge von der Gesellschaft ausbezahlte Vergütung könnte nach der jüngeren Rsp des BGH durchaus auch die Gefahr strafrechtlicher Sanktionen mit sich bringen (Mannesmann).

$\left.{ }^{119}\right)$ Hopt/M. Roth, $\mathrm{AktG}^{4} \& 114 \mathrm{Rz} 54$; Peltzer, ZIP 2007, 308

$\left.{ }^{120}\right)$ BGHZ 126, 345. Vgl Semler in MünchKomm AktG ${ }^{2}$ $\S 114 \mathrm{Rz} 78$.

$\left.{ }^{121}\right)$ Die Zustimmung des Aufsichtsrats zu einer Vergütung in Höhe des bisherigen Vorstandsbezugs erscheint per se fragwürdig, weil sie sich offensichtlich nicht primär am Wert der Beratungsleistung, sondern an den Einkunftsvorstellungen des Beraters (und Mehrheitsgesellschafters) orientiert; unbegründbar ist die Vergütung auch dann, wenn für die allein persönlich zu erbringende Beratungsleistung des Aufsichtsratsvorsitzenden der Kleinbank mehr bezahlt wird, als Vorstandsvorsitzende von Großbanken erhalten. Darüber hinaus erscheint in dem konkreten Fall kaum geklärt, ob die zu erbringenden Leistungen nicht bereits im Rahmen der Aufsichtsratstätigkeit geschuldet sind (womit eine zusätzliche Vergütung ausscheidet). Dem Wohl des Unternehmens scheint die hohe Vergütung prima facie nicht zu dienen. Vgl zu den Rechtsfolgen (nach deutschem Recht) Drygala in K. Schmidt/Lutter, AktG \& $113 \mathrm{Rz} 17 \mathrm{f}$.

${ }^{122}$ ) So jedenfalls die deutsche Rsp; vgl LG Stuttgart ZIP 1998,1279

${ }^{123}$ ) Kalss in Doralt/Nowotny/Kalss, AktG $\S 92 \mathrm{Rz} 58 \mathrm{f}$.

$\left.{ }^{124}\right)$ Dazu Kalss in Doralt/Nowotny/Kalss, AktG $\S 96$ $\mathrm{Rz}$ 92; W. Jud, Vorratsbeschlüsse im System zustimmungspflichtiger Geschäfte des Aufsichtsrats, ÖBA 1993, $773 \mathrm{ff}$

${ }^{125)}$ Dafür Fida, wbl 2006, 360 schäfts gefasst wurde und die Sonderinteressen uU nicht mehr transparent sind. Jedenfalls sind alle Voraussetzungen und Anforderungen, die für normale Beschlüsse gelten, auch für Vorratsbeschlüsse zu berücksichtigen (insb Informationstiefe und -dichte). Vertretbar dürften Vorratsbeschlüsse sein, soweit von der Anwendung von $\S 95$ Abs 5 Z 12 AktG im Konzern ausgegangen wird und es um diese geht ${ }^{126}$ ).

\section{Stimmverbot und Folgen für die Beschlussfä- higkeit des Aufsichtsrats}

Es stellt sich die Frage der Stimmberechtigung des betroffenen Aufsichtsratsmitglieds. Zwar würde auch bei Stimmberechtigung ein Teil des Regelungsziels verwirklicht (Information der übrigen Aufsichtsratsmitglieder). Doch eine Teilnahme des betroffenen Mitglieds an der Abstimmung ist mit dem zweiten Regelungsziel nicht vereinbar, nämlich der Kontrolle, die der Aufsichtsratsbeschluss für diese Geschäfte bringen soll. Das Mitglied selbst ist in dieser Hinsicht unzweifelhaft befangen und kann auch in Österreich nach allgemeinen Grundsätzen nicht mitstimmen ${ }^{127}$ ).

Auch in Österreich kann dadurch die Beschlussunfähigkeit eintreten. Anders als die deutsche Parallelbestimmung stellt $\S 92$ Abs 5 AktG allerdings für die Beschlussfähigkeit bloß auf die Teilnahme an der Sitzung (und nicht auf die Teilnahme an der Beschlussfassung) ab. Das Problem stellt sich also nach dem österr Gesetzeswortlaut weniger scharf: Die Teilnahme an der Sitzung ist möglich, selbst wenn an der Beschlussfassung (wegen Stimmverbots) nicht teilgenommen wird. Dennoch sprechen gute Gründe dafür, das vom Stimmrecht ausgeschlossene Mitglied auch von der Sitzung auszuschließen ${ }^{128}$ ), wodurch die Beschlussfähigkeit verloren gehen kann. In Abwägung der Regelungszwecke von $\S 92 \mathrm{Abs} 5 \mathrm{AktG}$ und $\S 95 \mathrm{Abs} 5$ Z 12 AktG bleibt wohl kein anderer praktikabler Lösungsweg als die Reduktion der erstgenannten Bestimmung.

\section{Sind Verträge mit einem Aufsichtsratsmitglied auch ohne Zustimmung des Aufsichtsrats wirk- sam?}

Ob Geschäfte, die unter $\S 95$ Abs 5 Z 12 AktG fallen, auch ohne Zustimmung des Aufsichtsrats wirksam zustande kommen, ist zweifelhaft. Die Frage ist unter Berücksichtigung der Grundsätze des Stellvertretungsrechts zu lösen.

${ }^{126}$ ) Denn im Konzern sind nur Teile des Gefahrenpotentials gegeben; regelmäßig geht es bei Konzerngeschäften nicht um die Gefährdung der Unabhängigkeit des ohnehin für die Muttergesellschaft tätigen Aufsichtsratsmitglieds oder um die Intransparenz gegenüber anderen Aufsichtsratsmitgliedern im Hinblick auf die evidenten Konzerngeschäfte. Zu Konzerngeschäften oben D.VIII.

${ }^{127}$ ) Kastner/Doralt/Nowotny, Gesellschaftsrecht ${ }^{5}, 243$; Kalss in Doralt/Nowotny/Kalss, AktG § 92 Rz 70 f; Poty$k a$, ÖJZ 2006, $195 \mathrm{FN} 24$.

${ }^{128}$ ) Lutter, FS Harm Peter Westermann 1172 FN 7. 


\section{Meinungsstand bei zustimmungspflichtigen Ge- schäften im Allgemeinen}

Der österr Gesetzgeber ordnet für alle in $\S 95$ Abs 5 AktG genannten Geschäfte an, dass sie nur mit Zustimmung des Aufsichtsrats erfolgen „sollen". Nach ganz unbestrittener Auffassung ist mit der Formulierung der Bestimmung als bloße „Sollensbestimmung“ nicht etwa eine sanktionslose Vorschrift mit bloßem Empfehlungscharakter gemeint. Es soll auch nicht in das Ermessen des Aufsichtsrats oder des Vorstands gestellt werden, ob über die Angelegenheit vom Aufsichtsrat entschieden wird ${ }^{129}$ ). Vielmehr ist ganz unbestritten, dass der Vorstand die zustimmungspflichtigen Geschäfte ohne Zustimmung des Aufsichtsrats nicht vornehmen darf und er bei Verletzung dieser Pflicht schadenersatzpflichtig werden kann und unter Umständen sogar einen Abberufungsgrund setzt. $\mathrm{Ob}$ die Geschäfte ohne Zustimmung im Außenverhältnis wirksam sind, lässt der Wortlaut des Gesetzes offen. Die hL ging bei den bisherigen zustimmungspflichtigen Geschäften nach $\S 95$ Abs 5 Z 1 - 11 AktG grundsätzlich von der Wirksamkeit im Außenverhältnis auch ohne entsprechenden Beschluss des Aufsichtsrats aus ${ }^{130}$ ). Begründet wird das meist nur mit der umfassenden Vertretungsmacht des Vorstands ( $§ 71$ Abs 1 AktG). Auf den ersten Blick mag auch die Wertung des $\S 25$ Abs 4 GmbHG diese Sichtweise stützen ${ }^{131}$ ): Nach der Bestimmung haften die Geschäftsführer bei In-sichGeschäften, die sie im eigenen oder fremden $\mathrm{Na}-$ men mit der Gesellschaft abschließen, wenn sie in Überschreitung ihrer Befugnisse zustimmungspflichtige Geschäfte vornehmen und die Gesellschaft dadurch einen Schaden erleidet.

Dass die Vertretungshandlungen trotz Überschreitung der Beschränkungen im Innenverhältnis nach außen stets wirksam sind, ist $\S 25 \mathrm{Abs} 4$ GmbHG allerdings nicht $\mathrm{zu}$ entnehmen. Die Bestimmung betrifft nur jene Fälle, in denen Schäden eintreten, weil die Vertretungshandlung der Geschäftsführer im konkreten Fall tatsächlich wirk-

129) Man kann das auch nicht etwa damit begründen, dass der österr Gesetzgeber anders als der deutsche in der heutigen Fassung von $\S 111$ Abs 4 S 2 dAktG die missverständliche Sollensbestimmung trotz vieler Novellierungen unverändert in der ursprünglichen Fassung des dAktG 1937 belassen hat. Denn in Anbetracht der völlig unbestrittenen Lehre und Praxis bestand zu einer Änderung kein Anlass. Nicht einmal eine Klarstellung schien bisher notwendig. S Kalss in Doralt/Nowotny/ Kalss, AktG $\& 88 \mathrm{Rz} 89 \mathrm{mwN}$.

$\left.{ }^{130}\right)$ Kastner, GesRZ 1982, 215; K. Wagner, Zu § 95 Abs 5 AktG und § 30j Abs 5 GmbHG, NZ 1983, 97 f; Reischauer, Gedanken zur Aufsichtsratszustimmung nach $\S 95$ Abs 5 AktG, FS Strasser II (1993) 288; Kastner/Doralt/Nowotny, Gesellschaftsrecht ${ }^{5}$, 261; Koppensteiner/Rüffler, $\mathrm{GmbHG}^{3} \S 30 \mathrm{j} \mathrm{Rz} 18$; Strasser in Jabornegg/Strasser, $\mathrm{AktG}^{4} \S \S 95-97 \mathrm{Rz} 41$; derselbe in Rummel (Hrsg), Kommentar zum Allgemeinen bürgerlichen Gesetzbuch ${ }^{3}$ (2000) §§ 1016, 1017 Rz 8; Krejci, Gesellschaftsrecht I (2005) 157

131) Den Hinweis auf diese Wertung im gegebenen $\mathrm{Zu}-$ sammenhang verdanke ich dem Begutachtungsverfahren. sam war. § 25 Abs 4 GmbHG ist also lediglich $\mathrm{zu}$ entnehmen, dass die Vertretungshandlung der Geschäftsführer im Außenverhältnis auch ohne $\mathrm{Zu}$ stimmung in manchen Fällen wirksam sein kann. $\S 25 \mathrm{Abs} 4 \mathrm{GmbHG}$ setzt also die Klärung dieser Frage im Einzelfall bereits voraus und ordnet eine Risikoverteilung bzw eine Sanktion für Fälle an, in denen die Gesellschaft durch die Überschreitung der Vertretungsbefugnisse der Geschäftsführer einen Schaden erlitten hat.

\section{Verkehrsschutz als maßgebliches Kriterium}

Jedenfalls bedarf die Annahme der hL, dass die Vertretungshandlungen des Vorstands im Außenverhältnis auch bei fehlender Zustimmung stets wirksam sind, einer stellvertretungsrechtlichen Vertiefung. Dass $\S 95$ Abs 5 AktG die Wirksamkeit im Außenverhältnis nicht berührt, ist keine Begründung, sondern ein Ergebnis ${ }^{132}$ ). Liest man die Gesetzesbestimmung unvoreingenommen, wäre auch die Annahme denkbar, dass die Wirksamkeit von Geschäften, die nur mit Zustimmung des Aufsichtsrats erfolgen sollen, eben von dieser Zustimmung abhängt (bzw die Vertretungshandlungen bei einem ablehnenden Beschluss endgültig unwirksam sind). Die Zustimmungserfordernisse des $\S 95$ Abs 5 AktG könnten bei einem solchen Verständnis als schlichte Einschränkung der Vertretungsmacht des Vorstands begriffen werden. Der bei der Ansicht der hL entstehende Widerspruch zwischen allgemeiner, umfassender Vertretungsmacht des Vorstands und Zustimmungserfordernis des Aufsichtsrats ließe sich damit vermeiden, und die zustimmungspflichtigen Geschäfte wären Ausnahmefälle von der umfassenden Vertretungsmacht des Vorstands.

Doch diese Sichtweise ist zu wenig differenziert und überzeugt nicht. Aus der gesetzlich angeordneten, unbeschränkten Vertretungsmacht des Vorstands ergibt sich zwar noch keine Lösung des Widerspruchs. Aber aus dem Zweck der unbeschränkten Vertretungsmacht und der historischen Entwicklung der Institution unbeschränkter Vertretungsbefugnisse im Handelsrecht kann ein Lösungsansatz abgeleitet werden. Der tragende Gedanke hinter der unbeschränkten und unbeschränkbaren Vertretungsmacht des Vorstands, aber auch der sonstigen Regeln über die Vertretung in Gesellschafts- und Unternehmensrecht ist der Verkehrsschutz. Dritte, die mit der Gesellschaft kontrahieren, sollen sich nicht erst über interne Vorgänge erkundigen müssen ${ }^{133}$ ): Der Verkehr soll

\footnotetext{
132) $\mathrm{S}$ aber die Nachweise zur hL in FN 130.

133) Dazu P. Bydlinski, Der sogenannte „Missbrauch“ unbeschränkbarer Vertretungsmacht, FS F. Bydlinski (2002) 34 mit umfassender Aufarbeitung der verschiedenen Strömungen der Rsp und der zahlreichen (widersprüchlichen und weitgehend unsystematischen) Ansätze der Lehre. Zur Rsp s etwa OGH 16.5.2000, 5 Ob 164/99z, ÖBA 2001, 242 mit Anm Bollenberger (für die Unwirksamkeit eines unrichtigen Grundbuchsauszugs bei Kenntnis der Unrichtigkeit); OGH 25.6.1996, 4 Ob 2078/
} 
und darf auf die Vertretungsbefugnis des Vorstands vertrauen. Ob der Verkehrsschutz (also das Vertrauendürfen des Geschäftsverkehrs auf die Vertretungsmacht des Vorstands) dort dasselbe Gewicht haben kann, wo das Gesetz (und nicht bloß die Satzung, eine interne Geschäftsordnung etc) Einschränkungen vorgibt, ist per se zweifelhaft. Bei Geschäften die nach $\S 95$ Abs 5 AktG nur mit Zustimmung des Aufsichtsrats erfolgen sollen, ist das Gewicht des Verkehrsschutzes geringer. Gänzlich zu vernachlässigen ist der Verkehrsschutz aber auch hier nicht, zumal der Katalog der zustimmungspflichtigen Geschäfte in vielfacher Hinsicht nicht abschließend ist. Die Satzung oder der Aufsichtsrat können bzw müssen den Katalog ergänzen bzw durch Wertgrenzen determinieren. Aber selbst die Detailkenntnis von Gesetzen fehlt im Geschäftsverkehr häufig ${ }^{134}$ ). Die Vertretungshandlungen des Vorstands im Außenverhältnis trotz fehlender Zustimmung des Aufsichtsrats stets als wirksam zu betrachten, wie es die hL annimmt, geht aber zu weit. Unter Berücksichtigung des Verkehrsschutzes als Ziel der unbeschränkten Vertretungsmacht und der entsprechenden stellvertretungsrechtlichen Grundsätze ergibt sich Folgendes:

\section{Kein Verkehrsschutz bei Kenntnis der Befugnis- überschreitung}

Verkehrsschutz ist kein Selbstzweck ${ }^{135}$ ). Wo Verkehrsschutz durch typisierte, unbeschränkte Vertretungsmacht bezweckt ist, müssen Dritte keine Nachforschungen über die Reichweite der Vertretungsbefugnis des Vertreters anstellen $\left.{ }^{136}\right)$. Bezweckt wird damit eine Vereinfachung des Geschäftsverkehrs.

96h, JBl 1997, 108 ff mit Anm Hügel 113 ff. Aus historischer und europarechtlicher Perspektive (Publizitätsrichtlinie) Auer, Missbrauch der Vertretungsmacht im Handels- und Gesellschaftsrecht, GesRZ 2000, 138 ff. Rechtsvergleichend Koziol, Risikoverteilung bei auftragswidrigem Handeln des Bevollmächtigten, FS Rey (2003) 427 ff; vgl Koppensteiner in Straube, $\mathrm{HGB}^{2} \S 126$ Rz 9; Thunhart, Eigenmächtige Vertragsabschlüsse des Bürgermeisters und die Notwendigkeit von Vertrauensschutz im Gemeinderecht, JBl 2001,78; Krejci, Die Kapitalgesellschaft als Spender und Förderer (II), GesRZ 1984, 150; anders allerdings Krejci, Unternehmensrecht ${ }^{4}$ (2008) 234, 240 und derselbe, Gesellschaftsrecht I 157 (kritisch dazu P. Bydlinski, FS F. Bydlinski 34 FN 59, 40). AA auch Jabornegg in Jabornegg (Hrsg), HGB Kommentar (1997) § $126 \mathrm{Rz} 11$. Umfassend aus deutscher Sicht G. Frotz, Verkehrsschutz und Vertretungsrecht (1972) $566 \mathrm{ff}$

${ }^{134}$ ) Vgl aber $\S 2$ ABGB, der zwar keine Fiktion der allgemeinen Gesetzeskenntnis aufstellt, jedoch klarstellt, dass Unkenntnis der Gesetzeslage keinen Einfluss auf die Wirksamkeit und Anwendbarkeit einer Norm hat. S F. Bydlinski in Rummel, $\mathrm{ABGB}^{3} \& 2 \mathrm{Rz} 1 \mathrm{ff} ;$ P. Bydlinski in Koziol/P. Bydlinski/Bollenberger (Hrsg), ABGB Kommentar $(\mathrm{KBB})^{2}(2007) \S 2 \mathrm{Rz} 1 \mathrm{ff}$.

$\left.{ }^{135}\right)$ P. Bydlinski, FS F. Bydlinski 35

$\left.{ }^{136}\right)$ P. Bydlinski, FS F. Bydlinski 34
Hingegen erscheint es zweifelhaft, Dritte und deren Vertrauen auf die Wirksamkeit der Vertretungsmacht zu schützen, wo kein Vertrauen gegeben ist. Jedenfalls kann von Vertrauensschutz nicht mehr die Rede sein, wenn geradezu die gegenteilige Situation vorliegt: Kenntnis der mangelnden Vertretungsbefugnis ${ }^{137}$ ). Wo Dritte positiv wissen, dass nur eine beschränkte Vertretungsmacht vorliegt, besteht kein Schutzbedürfnis eines wissenden Vertragspartners der Gesellschaft, denn Unkenntnis liegt keine vor ${ }^{138}$ ).

Konkret bedeutet das hier: Weiß das kontrahierende Aufsichtsratsmitglied vom Erfordernis, einen Genehmigungsbeschluss des Aufsichtsrats einzuholen, scheidet die Wirksamkeit der Vertretungshandlungen des Vorstands ohne Zustimmung des Aufsichtsrats bereits aus den genannten Gründen aus. Bei Kenntnis der Überschreitung von Befugnissen kann der Verkehrsschutz (in concreto: Schutz des Vertrauens des Aufsichtsratsmitglieds in die Gültigkeit des Geschäfts) nicht die Wirksamkeit des Geschäfts rechtfertigen ${ }^{139}$ ). Der Grund, der sonst für die Wirksamkeit des Geschäfts bei der streng typisierten Reichweite der Vertretungsmacht spricht, nämlich der Vertrauensschutz des Dritten im Geschäftsverkehr, fehlt $\left.{ }^{140}\right)$. Das Aufsichtsratsmitglied ist eben kein „Dritter" im üblichen Sinn.

\section{Was gilt bei (fahrlässiger) Unkenntnis der Be- fugnisüberschreitung?}

Fraglich erscheint die Lösung, wenn die Handelnden keine positive Kenntnis des Zustimmungserfordernisses hatten: Ein (ahnungsloses) Aufsichtsratsmitglied schließt mit der Gesellschaft einen Vertrag, ohne dass ein entsprechender Be-

$\left.{ }^{137}\right) \mathrm{Vgl}$ P. Bydlinski in $\mathrm{KBB}^{2} \S 1016 \mathrm{Rz} 1 \mathrm{ff}$, insb Rz 5 mit dem Hinweis auf die überraschende Uneinheitlichkeit der Lehre und Rsp.

${ }^{138}$ ) P. Bydlinski, FS F. Bydlinski 39; ihm folgend Apathy in Schwimann (Hrsg), ABGB Praxiskommentar ${ }^{3}$ IV (2006) $\S 1016 \mathrm{Rz} 13$. Anders etwa Strasser in Rummel, $\mathrm{ABGB}^{3}$ \& $\$ 1016$ f Rz 23 ff; Krejci, Unternehmensrecht ${ }^{4}$, 240, der Ungültigkeit erst dann annimmt, wenn Schädigungsabsicht des Vertreters im Zusammenwirken mit dem Dritten vorliegt, während Koziol/Bollenberger in Koziol/Welser, Bürgerliches Recht ${ }^{12}$ I (2002) 193 nur auf den Bewusstseinszustand des Dritten abstellen; ebenso nun auch Kletečka in Koziol/Welser, Bürgerliches Recht ${ }^{13}$ I (2006) 215 .

$\left.{ }^{139}\right)$ S das Beispiel von P. Bydlinski, FS F. Bydlinski 33.

$\left.{ }^{140}\right) \mathrm{Vgl}$ die Kritik an der hL von P. Bydlinski, FS F. Bydlinski 34: „Dem Dritten solle es möglich sein, sich aus den Interna der Gesellschaft herauszuhalten. Wer von den Interna Kenntnis hat, ist aber schon mitten drin! (Vor-)Vertragliche Nebenpflichten begegnen uns auch im Handelsrecht auf Schritt und Tritt. Und davon soll in Vertretungsfällen überhaupt keine Rede mehr sein; sogar dann nicht, wenn es dem Dritten klar sein muss, dass das Geschäft von seinem Vertragspartner aller Voraussicht nach so nicht gewünscht ist? Ein derart unausgewogenes Ergebnis kann weder mit Verkehrsschutz noch mit der Beschleunigung des Handelsverkehrs gerechtfertigt werden." 
schluss des Aufsichtsrats eingeholt wird. Selbst wenn mit der $\mathrm{hL}$ allgemein von der Wirksamkeit der Vertretungshandlungen des Vorstands nach Z 1 - 11 ohne Zustimmung des Aufsichtsrats ausgegangen wird, ist die Lösung bei Z 12 möglicherweise anders.

Zwar läge es auf den ersten Blick nahe, die erst später eingefügte $Z 12$ gleich zu behandeln und die wirksame Vertretung durch den Vorstand bei Unkenntnis auch ohne Zustimmung des Aufsichtsrats anzunehmen. Damit wäre $\S 95$ Abs $5 \mathrm{Z} 12$ AktG (dem Verständnis der hL folgend) eine bloß interne Regel der Gesellschaft und würde die unbeschränkte Vertretungsmacht des Vorstands im Außenverhältnis nicht berühren - der Vertragspartner müsste sich um die hier erörterten „Interna" der Gesellschaft nicht kümmern ${ }^{141}$ ).

Bei näherer Betrachtung zeigt sich allerdings, dass bei fehlender Genehmigung gem $\S 95$ Abs 5 Z 12 AktG im Vergleich zu den Fällen der Z 1 11 ein wesentlicher Unterschied besteht, der Auswirkung auf die Gültigkeit des genehmigungslosen Geschäftes haben muss. Denn bei den Geschäften, für die $\S 95$ Abs 5 AktG bereits vor dem GesRÄG 2005 eine Zustimmung verlangt hatte $(Z 1-11)$, geht es um Geschäfte mit Außenstehenden, die in aller Regel wegen ihrer Bedeutung und ihres Risikos für die Gesellschaft der Zustimmung des Aufsichtsrats bedürfen. Hingegen geht es bei Z 12 um ein Vertragsverhältnis zwischen der Gesellschaft und einem Aufsichtsratsmitglied, das nicht wegen der Bedeutung des Geschäfts für die Gesellschaft zustimmungspflichtig ist, sondern wegen der oben dargestellten Gründe: Interessenkollisionen und die Schwächung der Unabhängigkeit des Organs Aufsichtsrat sollen durch Transparenz und Kontrolle vermieden werden. Daraus ergibt sich folgender Unterschied: Im allgemeinen Geschäftsverkehr mag es gerechtfertigt sein, dass sich unbeteiligte Dritte nicht für die interne Beschränkung der Vertretungsmacht des Vorstands interessieren müssen, insb dass keine Nachforschungspflichten bestehen und dass im Ergebnis der Verkehrsschutz die Wirksamkeit der Vertretungshandlung im Außenverhältnis (unabhängig von Fehlern bei der gehörigen Willensbildung im Innenverhältnis) begründet. Für Geschäfte, bei denen der Vertragspartner der AG hingegen ein Aufsichtsratsmitglied ist, trägt diese Begründung nicht. Das gilt nicht nur für die Sachverhaltsfrage, ob die Zustimmung konkret erteilt wurde, sondern auch für die Rechtsfrage, ob das Geschäft zustimmungspflichtig ist. Denn anders als ein Dritter muss sich jedes Aufsichtsratsmitglied aufgrund seiner Organstellung über die gesetzlichen Regeln zu Geschäften der AG mit Aufsichtsratsmitgliedern informieren und auf deren korrekte Anwendung achten. Dazu gehört insb die Frage nach dem Erfordernis der Zustimmung des Aufsichtsrats zu Geschäften der

$\left.{ }^{141}\right)$ Allgemein dazu P. Bydlinski in $\mathrm{KBB}^{2} \S 1016 \mathrm{Rz} 5$; derselbe, FS F. Bydlinski 34; Krejci, Gesellschaftsrecht I 157 .
AG mit dem Aufsichtsratsmitglied. Es wäre absurd, ein Aufsichtsratsmitglied von dieser Pflicht $\mathrm{zu}$ befreien, weil es Vertragspartner der AG ist. Gerade im Falle eines drohenden Interessenkonflikts sind die entsprechenden Zustimmungserfordernisse zu beachten ${ }^{142}$ ). Für Verträge zwischen Gesellschaft und Aufsichtsratsmitglied gilt daher: Auch wenn das Aufsichtsratsmitglied nicht wusste, dass die Zustimmung des Aufsichtsrats fehlt, oder nicht wusste, dass die Zustimmung überhaupt erforderlich ist, kann der Verkehrsschutz nicht die Wirksamkeit der Vertretungshandlung des Vorstands begründen. Fehlt die Genehmigung des Aufsichtsrats, ist das Geschäft unwirksam.

Dieses Ergebnis wird durch ein weiteres Argument bekräftigt: Die Gefahr von Nachteilen für den Machtgeber gilt allgemein als eines der relevanten Kriterien bei der Eingrenzung der Reichweite wirksamer Vertretung ${ }^{143}$ ). Das Zustimmungserfordernis nach Z 12 besteht gerade wegen der geschilderten Gefahren bei diesen Geschäften, also wegen der möglichen Nachteile für den Machtgeber, hier die Gesellschaft. Insb droht die Schwächung der Funktionsfähigkeit des Aufsichtsrats, aber auch Missbrauch bei unangemessenem Entgelt $\left.{ }^{144}\right)$.

\section{Verträge mit Unternehmen, an denen ein Auf- sichtsratsmitglied ein erhebliches wirtschaftli- ches Interesse hat}

Bei ,Verträgen mit Unternehmen, an denen ein Aufsichtsratsmitglied ein erhebliches wirtschaftliches Interesse hat", könnte die Lage anders sein. Die eben behandelten rechtsgeschäftlichen Wertungen passen uneingeschränkt nur auf die Situation, in der das Aufsichtsratsmitglied selbst den Vertrag mit dem Vorstand abschließt. Wenn hingegen die Gesellschaft mit einem Unternehmen, an dem das Aufsichtsratsmitglied ein erhebliches wirtschaftliches Interesse hat, abschließt und dieses von jemand anderem als dem Aufsichtsratsmitglied vertreten ist, gilt Folgendes: Bei Kenntnis der

$\left.{ }^{142}\right)$ Vgl Flume, Allgemeiner Teil des Bürgerlichen Rechts ${ }^{3}$ II (1979) 790. Nach Flume kommt es dabei nicht darauf an, ob der Vertragspartner fahrlässig (oder grob fahrlässig) den Mangel der Vertretungsmacht nicht kannte, sondern darauf, ob dies „offenbar“ ist, ob „ein reasonable man ihn erkennen würde“. Da es hier nicht um eine rechtsgeschäftliche Beschränkung der Vollmacht, sondern um eine gesetzliche - im Aktiengesetz (und nicht in irgendeinem Nebengesetz) enthaltene - Regelung geht, kann auch nach dieser Sichtweise angenommen werden, dass der Mangel der Vertretungsmacht offenbar ist: Es gibt überhaupt nur sehr wenige gesetzliche Bestimmungen zu den Pflichten des Aufsichtsrats. Aufsichtsratsmitgliedern ist es durchaus zumutbar, sich mit den zentralen Rechtsgrundlagen ihrer Pflichten vertraut zu machen. Selbst wenn ein Aufsichtsratsmitglied von $\S 95$ Abs 5 Z 12 AktG im konkreten Fall nichts wusste, hätte es - in diesem Zusammenhang - davon wissen müssen.

$\left.{ }^{143}\right)$ Vgl P. Bydlinski, FS F. Bydlinski $24 \mathrm{ff}$.

${ }^{144}$ ) Vgl Vetter, ZIP 2008, 6 mwN aus der deutschen Rsp. 
Zustimmungserfordernisse des handelnden Vertreters greifen die bereits genannten, allgemeinen Bedenken gegen Verkehrs- und Vertrauensschutz ein. Ohne Zustimmung des Aufsichtsrats wird daher ein solches Geschäft nicht wirksam abgeschlossen.

Besteht keine Kenntnis der Situation und der Zustimmungserfordernisse, kann der Verkehrsschutz eingreifen, denn aktive Nachforschungspflichten bestehen für echte Außenstehende (hier das „Unternehmen“) nicht. Soweit das Aufsichtsratsmitglied in den Vertragsschluss nicht involviert ist und die Handelnden keine Kenntnis über die wirtschaftlichen Interessen des Aufsichtsratsmitglieds haben, fehlen auch die typischen Gefahren, denen $\S 95$ Abs 5 Z 12 AktG begegnen soll. Der Verkehrsschutz kann in dieser Fallgruppe zur Wirksamkeit der Vertretungshandlung im Außenverhältnis auch ohne Genehmigung des Aufsichtsrats führen. Insoweit besteht Gleichklang mit den anderen in $\S 95 \mathrm{Abs} 5 \mathrm{Z} 1$ bis $11 \mathrm{AktG}$ geregelten Fällen $\left.{ }^{145}\right)$.

\section{F. Unerledigte Probleme bei Arbeitnehmervertre- tern}

Die jüngere Vergangenheit zeigt, dass auch die Beeinflussung des Stimmverhaltens der Arbeitnehmervertreter durch Gewährung finanzieller Sondervorteile und geldwerter Leistungen vorkommt. Der aus Deutschland stammende Fall Volkswagen ${ }^{146}$ ) wird regelmäßig vor dem Hintergrund der besonders ausgeprägten deutschen Mitbestimmung diskutiert ${ }^{147}$ ). Doch auch in Österreich sind vergleichbare Probleme denkbar, obwohl sich die Mitbestimmung in wesentlichen Punkten von den deutschen Modellen ${ }^{148}$ ) unterscheidet. Soweit die Kapitalvertreter geschlossen agieren, besteht in der österr Praxis wegen der Drittelparität (anders als in Deutschland) kein Anreiz, Arbeitnehmervertreter durch Sondervorteile für sich zu gewinnen. Hingegen kann es auf deren Stimmen auch in Österreich ankommen, wenn Kapitalvertreter verschiedener Aktionärsgruppen im Aufsichtsrat gegenläufige Ansätze verfolgen. Dann entscheiden uU die Stimmen der Arbeitnehmervertreter.

Mit Sicherheit hatte der Gesetzgeber nicht primär auf die besondere Situation der Arbeitnehmervertreter gezielt, als $\S 95$ Abs 5 Z 12 AktG entworfen wurde (der Zusammenhang zu Arbeitnehmervertretern wurde - soweit ersichtlich - im Ge-

${ }^{145}$ ) Dazu gehört die mögliche schadenersatzrechtliche Haftung, wenn der Gesellschaft durch die nicht genehmigten Geschäfte ein Nachteil entsteht. UU kann in der genehmigungslosen Vornahme derartiger Geschäfte auch ein wichtiger Grund für eine vorzeitige Abberufung liegen

${ }^{146}$ ) Vgl zB Peitsmeier, VW Affäre, Was wußte Hartz?, FAZ 8. Juli 2005

${ }^{147}$ ) S etwa Rüthers, VW: Gemeinsamer Verrat an der Mitbestimmung?, NJW 2007, $195 \mathrm{ff}$

${ }^{148}$ ) Überblick über die verschiedenen Formen der Mitbestimmung bei Hüffer, $\mathrm{AktG}^{7} \S 95 \mathrm{Rz} 1 \mathrm{ff}$ und $\S 96 \mathrm{Rz} 1 \mathrm{ff}$, $\mathrm{Rz} 4 \mathrm{ff}$ setzgebungsprozess nirgendwo hergestellt). Das überrascht nicht, weil $\S 95$ Abs $5 \mathrm{Z} 12 \mathrm{AktG}$ für Sachverhalte geschaffen wurde, die typischerweise im Zusammenhang mit Kapitalvertretern und nicht mit Arbeitnehmervertretern zu erwarten sind.

Da die Gesetzeslage den Anwendungsbereich nicht einschränkt, unterliegen ihr wohl auch Geschäfte der Arbeitnehmervertreter mit der Gesellschaft. Die Gefährdung der unabhängigen Entscheidungsfindung besteht auch beim Arbeitnehmervertreter. So bedarf jede entgeltliche Beratung der Gesellschaft bzw des Vorstands durch einen Arbeitnehmervertreter entsprechend den erörterten Tatbestandselementen der Zustimmung des Aufsichtsrats. Insoweit besteht kein Unterschied $\mathrm{zu}$ Beratungsleistungen, die zB ein dem Aufsichtsrat angehöriger Rechtsanwalt erbringt.

In anderem Zusammenhang ergeben sich allerdings Besonderheiten für die Situation der Arbeitnehmervertreter: Ihre Aufsichtsratstätigkeit üben sie nach $\S 110$ ArbVG ehrenamtlich aus - nur für angemessene Barauslagen besteht ein Anspruch auf Ersatz. Nun besteht bereits bei der Festlegung des Ausmaßes erstattungsfähiger Kosten ein gewisser Spielraum. Aber auch die bisherigen Bezüge, die bei Freistellung weiterlaufen, und die Gestaltung des Dienstvertrages können im gegebenen Zusammenhang relevant sein. Das Dienstverhältnis beruht auf einem vor der Entsendung in den Aufsichtsrat geschlossenen Vertrag. Während bei Kapitalvertretern nach $\S 87$ Abs 1a AktG vor der Wahl in den Aufsichtsrat die Information der Hauptversammlung über Umstände verlangt wird, die auch nur die Besorgnis einer Befangenheit begründen könnten, fehlt eine vergleichbare Information bei Arbeitnehmervertretern. Bei Kapitalvertretern wären wohl gerade Dauerschuldverhältnisse mit der Gesellschaft offenzulegen. Die Informationspflicht hat bei Kapitalvertretern $\mathrm{mE}$ eine doppelte Funktion: Es soll die Wahl der Aufsichtsratsmitglieder durch die Hauptversammlung nur in Kenntnis der wesentlichen Eigenschaften der Kandidaten erfolgen ${ }^{149}$ ), und es soll die Transparenz bei Sonderinteressen gegenüber den anderen Aufsichtsratsmitgliedern hergestellt werden. Im Kontrast mag es als ein Mangel erscheinen, dass etwa Dienstverträge der Arbeitnehmervertreter mangels Anwendbarkeit von $\S 87$ Abs 1a AktG dem Aufsichtsrat nicht zur Kenntnis gebracht werden. Dieser Eindruck ist jedoch nicht richtig. Denn während bei einem Kapitalvertreter im Idealfall keine Umstände vorliegen sollten, die die Besorgnis einer Befangenheit begründen können, also keine anderen Vertragsbeziehungen zur Gesellschaft bestehen sollten, ist bei Arbeitnehmervertretern die Lage zwangsläufig anders. Sie müssen in einer Vertragsbeziehung zur Gesellschaft stehen. Während typische Dauerschuldver-

${ }^{149}$ ) Zu den Rechtsfolgen fehlerhafter oder unterbliebener Information der Hauptversammlung nach § 87 Abs 1a AktG und $\S 95$ Abs 5 Z 12 AktG s oben D.II. 
hältnisse eines in den Aufsichtsrat zu wählenden Kandidaten sicher offenzulegen sind, ist eine vergleichbare Annahme für die Dienstverträge der Arbeitnehmervertreter nicht überzeugend. Die Gefahren der Manipulation bzw Einflussnahme sind bei einem vor Eintritt in den Aufsichtsrat geschlossenen Dienstvertrag nur schwer vorstellbar. $\S 95$ Abs 5 Z 12 AktG ist damit auf Dienstverträge der Arbeitnehmervertreter nicht anzuwenden.

Anders ist die Lage hingegen bei neu hinzukommenden, also nach der Entsendung in den Aufsichtsrat gewährten Vergünstigungen. Insb sind dabei neben einer direkten Erhöhung der Bezüge Sachzuwendungen (zB Dienstauto) denkbar. Bei derartigen zusätzlichen Leistungen kann durchaus die Einflussnahme auf die Entscheidungsfindung bezweckt sein. Deshalb sind solche Zuwendungen als Leistungen im Sinne von $\S 95$ Abs 5 Z 12 AktG zu qualifizieren ${ }^{150}$ ). Ob eine Verpflichtung, wie es der Gesetzestext fordert, gegenüber der Gesellschaft begründet wird, ist aber zweifelhaft. Die Formulierung des Gesetzestextes passt nicht uneingeschränkt auf die Situation, wobei zu bedenken ist, dass der Gesetzgeber diese Frage wohl nicht vor Augen hatte. Aber auch wenn keine Verpflichtung des Arbeitnehmervertreters zu einer Leistung begründet wird, spricht ein klares argumentum a minori ad maius für die Anwendung von $\S 95$ Abs 5 Z 12 AktG: Sogar bei einer adäquaten Gegenleistungsverpflichtung des Aufsichtsratsmitglieds ist ein Beschluss des Gremiums zur Genehmigung erforderlich. Erst recht ist daher anzunehmen, dass der Aufsichtsrat informiert werden sollte und seine Zustimmung einzuholen ist, wenn das begünstigte Aufsichtsratsmitglied sich für eine Zuwendung zu keiner konkreten Gegenleistung gegenüber der Gesellschaft verpflichtet.

${ }^{150)}$ Dazu bei D.VII.
Problematisch sind darüber hinaus jene Situationen, bei denen Arbeitnehmervertretern ohne spezifische vertragliche Grundlage Sondervorteile, insb Sachzuwendungen, gewährt werden.

De lege ferenda wäre eine klarstellende Regelung zur Wahrung der Unabhängigkeit der Arbeitnehmervertreter gegenüber dem Vorstand zu erwägen. Dem Aufsichtsrat sollten $\mathrm{mE}$ aber schon nach der geltenden Rechtslage Änderungen der Entlohnung der Arbeitnehmervertreter zur Genehmigung vorgelegt werden. Gleiches gilt für vertraglich vereinbarte oder bloß faktisch gewährte Sachzuwendungen. Die Information müsste ähnlichen Anforderungen genügen wie jene bei Geschäften von Kapitalvertretern mit der Gesellschaft.

\section{G. Ausblick}

Mit der Einführung von $\S 95$ Abs 5 Z 12 AktG hat der Gesetzgeber einen weitreichenden Schritt gesetzt, um Interessenkonflikte von Kapitalvertretern im Aufsichtsrat zu vermeiden. Die Verbesserungen der Kontrolle und Transparenz werden allerdings auch die Zusammensetzung von Aufsichtsräten beeinflussen. Für Aufsichtsratsmitglieder, die den beratenden Berufen angehören, dürfte die Annahme eines Aufsichtsratssitzes weniger attraktiv als bisher werden: Die Beratung der Gesellschaft durch das Aufsichtsratsmitglied oder dessen Kanzleipartner ist nicht mehr ohne weiteres möglich. Steigen die im internationalen Vergleich niedrigen Aufsichtsratsvergütungen nicht an, dürfte es schwieriger werden, Rechtsanwälte, Notare, Steuerberater oder Wirtschaftsprüfer, deren Expertise von den Gesellschaftern geschätzt wird, für ein Aufsichtsratsmandat zu gewinnen.

Korrespondenz: Dr. Walter Doralt, Referent am MaxPlanck-Institut für ausländisches und internationales Privatrecht, Mittelweg 187, D-20148 Hamburg, Deutschland; e-mail: doralt@mpipriv.de. 